\title{
The Condorcet Paradox Revisited
}

Citation for published version (APA):

Herings, P. J-J., \& Houba, H. (2016). The Condorcet Paradox Revisited. Social Choice and Welfare, 47(1), 141-186. https://doi.org/10.1007/s00355-016-0950-7

Document status and date:

Published: 01/06/2016

DOI:

10.1007/s00355-016-0950-7

Document Version:

Publisher's PDF, also known as Version of record

\section{Please check the document version of this publication:}

- A submitted manuscript is the version of the article upon submission and before peer-review. There can be important differences between the submitted version and the official published version of record.

People interested in the research are advised to contact the author for the final version of the publication, or visit the DOI to the publisher's website.

- The final author version and the galley proof are versions of the publication after peer review.

- The final published version features the final layout of the paper including the volume, issue and page numbers.

Link to publication

\footnotetext{
General rights rights.

- You may freely distribute the URL identifying the publication in the public portal. please follow below link for the End User Agreement:

www.umlib.nl/taverne-license

Take down policy

If you believe that this document breaches copyright please contact us at:

repository@maastrichtuniversity.nl

providing details and we will investigate your claim.
}

Copyright and moral rights for the publications made accessible in the public portal are retained by the authors and/or other copyright owners and it is a condition of accessing publications that users recognise and abide by the legal requirements associated with these

- Users may download and print one copy of any publication from the public portal for the purpose of private study or research.

- You may not further distribute the material or use it for any profit-making activity or commercial gain

If the publication is distributed under the terms of Article $25 \mathrm{fa}$ of the Dutch Copyright Act, indicated by the "Taverne" license above, 


\title{
The Condorcet paradox revisited
}

\author{
P. Jean-Jacques Herings ${ }^{1}{ }_{(\mathbb{C}} \cdot$ Harold Houba $^{2}$
}

Received: 23 May 2014 / Accepted: 31 January 2016 / Published online: 16 March 2016 (C) The Author(s) 2016. This article is published with open access at Springerlink.com

\begin{abstract}
We analyze the Condorcet paradox within a strategic bargaining model with majority voting, exogenous recognition probabilities, and no discounting for the case with three players and three alternatives. Stationary subgame perfect equilibria (SSPE) exist whenever the geometric mean of the players' risk coefficients, ratios of utility differences between alternatives, is at most one. SSPEs ensure agreement within finite expected time. For generic parameter values, SSPEs are unique and exclude Condorcet cycles. In an SSPE, at least two players propose their best alternative and at most one player proposes his middle alternative with positive probability. Players never reject best alternatives, may reject middle alternatives with positive probability, and reject worst alternatives. Recognition probabilities represent bargaining power and drive expected delay. Irrespective of utilities, no delay occurs for suitable distributions of bargaining power, whereas expected delay goes to infinity in the limit where one player holds all bargaining power. An increase in the recognition probability of a player may
\end{abstract}

The authors thank János Flesch, Eilon Solan, Frans Spinnewyn, Peter Sudhölter, and Walter Trockel for valuable comments.

P. J.-J. Herings would like to thank the Netherlands Organisation for Scientific Research (NWO) for financial support.

$凶 \quad$ P. Jean-Jacques Herings

p.herings@maastrichtuniversity.nl

Harold Houba

harold.houba@vu.nl

1 Department of Economics, Maastricht University, P.O. Box 616, 6200 MD Maastricht, The Netherlands

2 Department of Econometrics and Tinbergen Institute, VU University Amsterdam, De Boelelaan 1105, 1081 HV Amsterdam, The Netherlands 
weaken his bargaining position. A player weakly improves his bargaining position when his risk coefficient decreases.

\section{Introduction}

Decisions on collective choice problems are often taken by means of majority voting, and the analysis of majority voting is therefore an important topic in political economy. When preferences are such that some alternative beats every other feasible alternative in a pairwise vote, i.e. there is a Condorcet winner, then this will be the outcome reached for a huge variety of games that capture the underlying institution. Such would be the case for instance in models with real-time agenda setting and fixed defaults as in Banks and Duggan (2000), in models with evolving defaults as studied in Bernheim et al. (2006), as well as in the more traditional social choice approach.

Unfortunately, Condorcet winners may not exist and this gives rise to the Condorcet paradox in which any alternative can be reached from any other by a sequence of alternatives, where each alternative in the sequence beats the previous one by a pairwise majority vote as has been demonstrated in McKelvey (1976, 1979). It has been shown in the literature that the occurrence of the Condorcet paradox is not an artifact. Work by Plott (1967), Rubinstein (1979), Schofield (1983), Cox (1984), and Breton (1987) shows that this paradox occurs generically.

The lack of Condorcet winners is also a frequently observed empirical phenomenon. Balinski and Laraki (2010) provide a detailed documentation of the occurrence of the Condorcet paradox in the 1976 Cabernet-Sauvignon wine tasting in Paris, the 1994 general election of the Danish Folketing, and the 2007 French presidential election. Roessler et al. (2013) explain the underdevelopment of the Roman metro system as a consequence of a Condorcet cycle in the majority preferences over building a metro, preserving antiquities, and not digging.

In its most simple form the paradox features three players, three alternatives, and players' preferences such that a pairwise vote over the alternatives results in a Condorcet cycle: one pair of players prefers the second alternative to the third alternative, another pair of players prefers the first alternative to the second alternative, and a third pair of players prefers the third alternative to the first alternative. Whether and how players reach an agreement in this case is an open issue. It is the main research question addressed in this paper.

We take the strategic bargaining approach to analyze the Condorcet paradox, an approach that is advocated in Baron and Ferejohn (1989) and Banks and Duggan (2000) to study collective decision problems and that extends the seminal work on bargaining by Rubinstein (1982) and Binmore (1987). Such an approach makes explicit how alternatives that are up for voting are selected and how players vote on alternatives, both on and off the equilibrium path. Our analysis complements the one of Baron and Ferejohn (1989), who use this bargaining protocol to examine the collective decision problem of dividing a surplus, or the more general framework of coalitional bargaining in Chatterjee et al. (1993), which is the relevant case when the players can make arbitrary side-payments and have utility functions that are linear in the side-payment received. 
In every bargaining round, exogenous and positive recognition probabilities select one player who has the right to propose. This recognized player either proposes one of the three alternatives or gives up the right to propose in which case the bargaining proceeds to the next round. In the former case, the other players publicly vote in a sequential order. Majority voting among three players implies that one vote in favor suffices for acceptance, after which the alternative will be implemented, and players receive their utility. Otherwise, no alternative is implemented and we proceed to the next round where random selection determines the next proposer. Perpetual disagreement leads to a utility of zero for every player. We are interested in the case where bargaining occurs relatively fast, so where players do not heavily discount the future. To analyze this case, we derive equilibria for the limit case where players do not discount the future at all.

We characterize the set of stationary subgame perfect equilibria (SSPE). A subgame perfect equilibrium is said to be stationary if the strategy of a player is the same whenever the player faces the same continuation game. In identifying identical continuation games, we follow the approach suggested in Maskin and Tirole (2001) for determining the notion of a stationary strategy. For a foundation of stationary equilibria, we refer to Bhaskar et al. (2013).

When a player proposes his middle or worst alternative, it will be accepted for sure by the player for whom this is the best alternative. Since proposing his middle alternative strictly dominates proposing his worst alternative, a player will never propose his worst alternative in an SSPE, and the SSPE utility of a player conditional on being the proposer weakly exceeds the utility of his middle alternative. When a player proposes his best alternative, it may or it may not be accepted by the player for whom this is the middle alternative, and it will be rejected by the player for whom this is the worst alternative. A proposing player thereby effectively faces a trade-off between getting the utility of his middle alternative for sure and proposing his best alternative, which may result in a rejection and thereby ultimately in the continuation probability distribution on alternatives.

We show that the continuation utility of a player is at most equal to the utility of his middle alternative, from which it follows that there is an advantage to propose. This implies that, except for degenerate cases, a player is never willing to give up his right to propose. Similarly, a player responding to a proposal consisting of his middle alternative may accept it, thereby securing the utility of his middle alternative, or may reject it, ultimately leading to the continuation probability distribution on all the alternatives.

We define an equilibrium type by the number of players that propose their best alternative for sure, as well as the number of players that accept their middle alternative for sure. We show that across all parameter values seven equilibrium types are possible, three of which occur for a degenerate set of parameter values only, leaving four generic equilibrium types.

Our main results are as follows.

Existence We derive a very simple condition that is necessary and sufficient for the existence of an SSPE in mixed strategy profiles. To express this condition, we define a player's risk coefficient as the ratio of the utility difference between his best and middle alternative to the utility difference between his middle and worst alternative. 
The risk coefficient of a player is less than or equal to one if and only if the player prefers his middle alternative to the fair lottery over his best and worst alternative. Risk coefficients are equal to a particular transformation of the risk limit of Zeuthen (1930) and Harsanyi (1977). The condition for existence states that the geometric mean of the players' risk coefficients should be less than or equal to one. As a side result, we also identify the smaller subclass of preferences for which pure strategy SSPEs exist.

Generic uniqueness For generic parameter values, SSPE utilities are unique, though in degenerate cases multiple SSPE giving rise to different utilities may co-exist.

Delay depends crucially on the division of bargaining power In bargaining models a suitable way to express bargaining power is by the choice of recognition probabilities, where more bargaining power corresponds to a higher recognition probability. The division of bargaining power is a key factor to explain expected bargaining delay. For each specification of the agents' utility functions it is possible to divide bargaining power in such a way that no delay occurs at all. At the same time, when almost all the bargaining power goes to a single agent, expected delay goes to infinity.

Stochastic cycles Infinite cycles occur according to the logic of the Condorcet paradox by assumption. However, within a cooperative game theoretic setting, Chwe (1994) argues that cycles cannot occur when players are farsighted. We study SSPE cycles in the sense of whether there is a positive probability that an equilibrium path can result in which all three alternatives have been proposed and rejected before some alternative is accepted. Generically, such SSPE cycles do not occur, though SSPE cycles are possible in degenerate cases.

Higher recognition probabilities may lead to a worse bargaining position We investigate the comparative statics on outcome probabilities induced by changes in the recognition probabilities in our discrete choice model featuring the Condorcet paradox. We consider the case where an increase in the recognition probability of a player leads to a proportional decrease in the recognition probability of the other two players. We say that a player's bargaining position improves if the probability of receiving his best alternative increases and the probability of receiving his worst alternative decreases. Restricting attention to the generic parameter values for which there is a unique SSPE, we find that increasing a player's recognition probability either improves his bargaining position, or has no effect at all, or worsens his bargaining position.

Lower risk coefficients lead to a weakly improved bargaining position We investigate the comparative statics on outcome probabilities induced by changes in the risk coefficients. Restricting attention to the generic parameter values for which there is a unique SSPE, we find that decreasing a player's risk coefficient either has no effect at all or leads to a higher probability of attaining his best alternative and a lower probability of attaining his worst alternative, so improves the player's bargaining position.

Since our equilibria will be described as solutions to a finite number of equations in the same number of unknowns, for generic values of our parameters, one can apply the implicit function theorem to derive equilibria nearby the SSPE with discount factor equal to one as a function of the discount factor. It follows that results regarding comparative statics of equilibria carry over to discount factors close to one. SSPE existence is not an issue for values of the discount factor below one. In the presence of discounting, equilibrium existence follows from standard results on equilibrium existence in stochastic games, see Fink (1964), Takahashi (1964), and Sobel (1971). 
For this class of games, Haller and Lagunoff (2000) show that the set of stationary equilibria is generically finite. Herings and Peeters (2004) show that generically there is an odd number of stationary equilibria.

For parameter values where the geometric mean of the players' risk coefficients is less than or equal to one, consider a sequence of discount factors tending to one from below. Then there is a corresponding sequence of SSPEs converging to the equilibrium when the discount factor is equal to one, which implies that the probability of reaching an agreement in a given bargaining round is bounded away from zero when the discount factor tends to one. On the other hand, for parameter values where the geometric mean of the players' risk coefficients is above one, there is no such sequence of SSPEs, and the probability of reaching an agreement in a given bargaining round tends to zero when the discount factor tends to one. Herings and Houba (2015) provides a detailed analysis of the relationship between SSPEs for high discount factors and the limit equilibrium.

Our properties regarding the existence of equilibrium and the occurrence of delay in an SSPE depend also crucially on our assumption that collective decision making concerns the choice out of a finite set of alternatives. In the case studied by Baron and Ferejohn (1989), the collective decision problem of dividing a surplus of size one and discount factors below one, there is essentially a unique SSPE and this equilibrium does not involve delay as has been shown by Eraslan (2002) and Eraslan and McLennan (2013). Banks and Duggan (2000) generalize the set-up of Baron and Ferejohn (1989) in several directions, and study collective decision making on a non-empty, compact and convex set of alternatives. They also include an analysis of the case where all discount factors are equal to one. The condition of limited shared weak preferences (LWSP) says that if an alternative distinct from some given alternative is weakly preferred to the given alternative by all members of an arbitrary coalition, then it can be approximated by alternatives that all members of the coalition strictly prefer to the given alternative. The model of Baron and Ferejohn (1989) satisfies LWSP. Banks and Duggan (2000) show that LWSP is sufficient for the existence of an SSPE without delay in the case with a non-empty, compact and convex set of alternatives, even when all discount factors are equal to one.

Apart from offering insights in collective choice problems, our model also applies to coalition and network formation, and thereby to marriage and roommate problems. Proposing an alternative corresponds to proposing a coalition in a coalition formation context and to proposing a link in a network formation model. Bloch (1996) studies a sequential game of coalition formation when the division of the coalitional surplus is fixed and payoffs are defined relative to the whole coalition structure. Bloch (1996) shows for the rejector-proposes protocol introduced in Selten (1981) that core stable coalition structures can be attained as a stationary subgame perfect equilibrium of the game, but that stationary subgame perfect equilibria in pure strategies may fail to exist when the condition of core stability is violated. When coalitional externalities are absent, one obtains the class of hedonic games studied in Bloch and Diamantoudi (2011). They note that, in roommate problems with odd top rings, equilibria in pure strategies do not exist. When interpreted as a game of coalition formation, our model allows for three non-trivial coalition structures to form, and the Condorcet cycle in our model is equivalent to the absence of a core stable coalition structure and the presence of an odd top ring. 
The paper is organized as follows. Section 2 describes the bargaining model. Section 3 analyzes the case where all players are symmetric. Section 4 introduces the notion of SSPE and characterizes the set of SSPEs as the solutions to a specific system of equations. In that section, we also derive some of the general properties and reduce the complexity of the problem at hand. Then, Sect. 5 analyzes this system by summarizing the various equilibrium types discussed before. The details of the calculations are relegated to Appendix A. All the other proofs can be found in Appendix B. Section 6 combines all the results of Sect. 5 and studies the questions of SSPE existence and uniqueness. Section 7 analyzes the potential for delay and cycles and Sect. 8 the comparative statics on outcome probability vectors induced by changes in recognition probabilities and risk coefficients. Section 9 concludes.

\section{The model}

Three players, labeled $i=1,2,3$, have to decide which out of three possible alternatives, $x_{1}, x_{2}$, and $x_{3}$, should be implemented. The preferences of the players satisfy the following restriction

$$
x_{1} \succ^{1,3} x_{2} \succ^{1,2} x_{3} \succ^{2,3} x_{1}
$$

The formulation in (2.1) means that players 1 and 3 prefer the alternative $x_{1}$ to $x_{2}$, players 1 and 2 prefer the alternative $x_{2}$ to $x_{3}$, and players 2 and 3 prefer the alternative $x_{3}$ to $x_{1}$, so the players are involved in a decision problem that gives rise to the Condorcet paradox. A naive approach would lead to the claim that majority voting over the alternatives results in a cycle.

Here we model majority voting over the alternatives by means of an explicit extensive-form game. We take the standard non-cooperative bargaining model from the literature, based on the work by Rubinstein (1982) and in particular Binmore (1987). The same bargaining protocol has been advocated in Banks and Duggan (2000) to analyze collective choice problems, and has been used in their work on bargaining in legislatures by Baron and Ferejohn (1989).

We assume that in each period $t$ some player, say player $i$, is selected randomly according to an a priori specified probability distribution. Player $i$ then decides either to make a proposal to the other two players, i.e. he proposes some alternative $x_{j}$, or he decides not to make a proposal, and the players reach period $t+1$. In the latter case, we say that player $i$ makes proposal $x_{0}$. In the former case, the other two players vote sequentially. ${ }^{1}$ To avoid inessential multiplicity of equilibria, we assume that the player who ranks the alternative highest, is the first one to vote. ${ }^{2}$

\footnotetext{
1 Simultaneous voting may lead to undesirable equilibria due to coordination failures. For instance, the case where all players vote in favor of all proposals leads to an equilibrium, as there is no player who can gain by deviating. To avoid this problem, it is standard to assume either sequential voting or simultaneous voting with players using stage-undominated voting strategies.

2 Suppose player 1 proposes $x_{2}$, the best alternative for player 2, and suppose that player 3 votes before player 2. The alternative $x_{2}$ is the worst alternative for player 3. Player 3 may nevertheless decide to vote Footnote 2 continued
} 
Table 1 The order of voting

\begin{tabular}{llllll}
\hline Proposal & Sequence & Proposal & Sequence & Proposal & Sequence \\
\hline$\left(x_{1}, 1\right)$ & $(3,2)$ & $\left(x_{1}, 2\right)$ & $(1,3)$ & $\left(x_{1}, 3\right)$ & $(1,2)$ \\
$\left(x_{2}, 1\right)$ & $(2,3)$ & $\left(x_{2}, 2\right)$ & $(1,3)$ & $\left(x_{2}, 3\right)$ & $(2,1)$ \\
$\left(x_{3}, 1\right)$ & $(3,2)$ & $\left(x_{3}, 2\right)$ & $(3,1)$ & $\left(x_{3}, 3\right)$ & $(2,1)$ \\
\hline
\end{tabular}

Table 1 illustrates the order in which players vote given a proposal by some player, where in the table $\left(x_{j}, i\right)$ means that proposal $x_{j}$ is made by player $i$. If player 1 proposes $x_{1}$, then we assume that first player 3 votes and next, conditional on a vote against by player 3 , player 2 . After player $i$ makes proposal $x_{j}$, the first player to respond is denoted by $f_{j i}$, the second by $s_{j i}$.

A voter casts a vote either in favor or against $x_{j}$. If the first voter casts a vote in favor of $x_{j}$, then together with the proposer he forms a majority in favor of $x_{j}$, the alternative $x_{j}$ is accepted, and bargaining ends. If the first voter votes against $x_{j}$, then the second voter is allowed to vote. If the second voter casts a vote in favor of $x_{j}$, then again a majority is in favor of $x_{j}$, the alternative $x_{j}$ is accepted, and bargaining ends. Otherwise, period $t+1$ is reached. In period $t+1 \mathrm{a}$ new proposer is selected, and the entire procedure is repeated.

We assume that the probability of being recognized as a proposer is given by $\rho=\left(\rho_{1}, \rho_{2}, \rho_{3}\right)$ in each period $t$, where $\rho_{1}+\rho_{2}+\rho_{3}=1$ and $\rho_{i}>0$ is the probability that player $i$ is recognized.

The preferences of the players are represented by von-Neumann Morgenstern utility functions. We normalize utilities in such a way that the utility of disagreement forever is 0 for all players.

We are interested in the case where bargaining occurs relatively fast, so players do not heavily discount the future. To analyze this case, we derive equilibrium for the limit case where players do not discount the future at all. Player $i$ 's utility of acceptance of proposal $x_{j}$ in period $t$ is equal to $u^{i}\left(x_{j}\right)$. To satisfy (2.1), we have that

$$
\begin{aligned}
& u^{1}\left(x_{1}\right)>u^{1}\left(x_{2}\right)>u^{1}\left(x_{3}\right) \geq 0, \\
& u^{2}\left(x_{2}\right)>u^{2}\left(x_{3}\right)>u^{2}\left(x_{1}\right) \geq 0, \\
& u^{3}\left(x_{3}\right)>u^{3}\left(x_{1}\right)>u^{3}\left(x_{2}\right) \geq 0 .
\end{aligned}
$$

For $i=1,2,3$, and $j=0,1,2,3$, we define $u_{j}^{i}=u^{i}\left(x_{j}\right), u_{j}=\left(u_{j}^{1}, u_{j}^{2}, u_{j}^{3}\right)$, $u^{i}=\left(u_{0}^{i}, u_{1}^{i}, u_{2}^{i}, u_{3}^{i}\right)^{\top}$, and $u=\left(u^{1}, u^{2}, u^{3}\right)$. For $i=1,2,3$, we define $b_{i}, m_{i}$, and $w_{i}$ as the number of the alternative related to the best, middle, and worst alternative for player $i$. For instance, we have $b_{1}=1, m_{2}=3$, and $w_{3}=2$.

Each sequence of proposers, proposals, and votes defines a history. A pure behavioral strategy of a player assigns an action to each history where he has to take a decision. Mixed behavioral strategies are defined in the usual way. Every strat-

in favor of $x_{2}$ since he knows that the proposal will be accepted anyhow by player 2 next and is therefore indifferent as far as his own voting behavior is concerned. 
egy implies a probability distribution $\left(\pi_{0}, \pi_{1}, \pi_{2}, \pi_{3}\right)$ over the four possible final outcomes, being perpetual disagreement, agreement on $x_{1}$, agreement on $x_{2}$, and agreement on $x_{3}$. Any mixed strategy therefore implies expected payoffs that are a weighted average of $u_{j}, j=0,1,2,3$, with weights $\pi_{j}$. Note that $\pi_{0}>0$ implies a positive probability of the players' worst possible outcome of perpetual disagreement.

Utility functions $u$ and recognition probabilities $\rho$ satisfying (2.2)-(2.4) determine a game $G=(u, \rho)$ in extensive form. The class of all such games is denoted $\mathcal{G}$.

\section{The symmetric case}

Throughout this section, we consider a special case of interest in more detail, namely what we call the symmetric case. In the symmetric case it holds that $\rho_{1}=\rho_{2}=$ $\rho_{3}=1 / 3$, and for $i=1,2,3$, the utilities $u_{b_{i}}^{i}, u_{m_{i}}^{i}$, and $u_{w_{i}}^{i}$ are independent of $i$. To simplify the analysis further, we assume $u_{w_{i}}^{i}=0$ for every player $i$. We normalize the utility of the best alternative to be equal to 1 . The utility of the middle alternative is independent of $i$ and is denoted by $c \in(0,1)$.

Assume players use symmetric strategies that are time and history independent. We use $p_{b}, p_{m}$, and $p_{w}$ to denote the probability that, conditionally on being recognized, a player proposes his best, middle, and worst alternative, respectively. For the moment, we ignore the possibility that a player gives up the right to propose, so $p_{b}+p_{m}+$ $p_{w}=1$. We use $a_{b}, a_{m}$, and $a_{w}$ to denote the probability that a player accepts his best, middle, and worst alternative, respectively, when responding to a proposal, so $0 \leq a_{b} \leq 1,0 \leq a_{m} \leq 1$, and $0 \leq a_{w} \leq 1$. Since we are looking at symmetric strategies, equilibrium utility is at most equal to $(1+c) / 3$ for every player. If a player proposes his middle alternative, he is sure it will be accepted by the player for whom this is the best alternative. It follows that conditional on being the proposer, the payoff of a player is at least $c$. It is then clear that no player would like to propose his worst alternative, since it would be accepted by the player for whom this is the best alternative, leading to a payoff of 0 . On the other hand, it is not a priori clear what happens if a player proposes his best alternative. For sure it will be rejected by the player for whom this is the worst alternative. However, the player for whom this is the middle alternative may or may not accept it. In conclusion, equilibria should have the property that $p_{w}=0, a_{b}=1$, and $a_{w}=0$. The only variables which are not yet determined are $p_{b}$ and $a_{m}$. The value of $p_{m}$ is determined by the fact that $p_{b}+p_{m}=1$.

We first consider the case with $c \in(1 / 2,1)$. We claim, and prove formally later on in Theorem A.4.4, that the pure strategy profile following from always proposing the best alternative and always accepting the middle alternative, so $p_{b}=1$ and $a_{m}=1$, is an equilibrium. The equilibrium does not involve delay. Clearly, in a symmetric equilibrium all three alternatives are selected with equal probability. Since there is immediate agreement at equilibrium, it holds that $\pi_{0}=0$ and $\pi_{1}=\pi_{2}=\pi_{3}=1 / 3$. The expected equilibrium utility is equal to $(1+c+0) / 3$, which is less than the utility $c$ of the middle alternative. The fact that the utility of the middle alternative is fairly high, i.e. above $1 / 2$, makes players willing to compromise and avoid equilibrium delay. 
To prove that $p_{b}=1$ and $a_{m}=1$ induces an equilibrium, we consider one-stage deviations and show that they are not profitable. We will show later on that the absence of profitable one-shot deviations implies the absence of profitable general deviations. When a player is selected as a proposer, he obtains a utility of 1 and clearly has no incentive to deviate. When a player has to vote on his middle alternative, he gets a utility of $c$ when he follows the equilibrium strategy. A one-shot deviation to a rejection leads to a uniform probability distribution on each outcome, giving rise to an expected utility of $(1+c) / 3$, which is less than $c$, so is not attractive. It will follow from Theorem A.4.4 that there are no other equilibria with proposer-independent acceptance probabilities. When acceptance probabilities are allowed to depend on the identity of the proposer, there are also other equilibria. For instance, in the off-the-equilibriumpath subgame where a player has proposed his worst alternative, the player for whom this is the best alternative votes first and might reject the proposal in the knowledge that the player voting after him will accept his middle alternative. This construction can also be used to generate non-symmetric equilibria. In Theorem 4.4 it will be shown that as far as equilibrium utilities are concerned, there is no loss of generality to restrict attention to proposer-independent acceptance probabilities. It now follows that equilibrium utilities are unique when $c \in(1 / 2,1)$.

We next move to the case where $c=1 / 2$. Although this case is clearly degenerate, it may exhibit quite different behavior in equilibrium. First of all, it follows from exactly the same argument as in the preceding paragraph that always proposing the best alternative and always accepting the middle alternative, $p_{b}=1$ and $a_{m}=1$, is an equilibrium that does not involve delay. Equilibrium utility is equal to $1 / 2$ for every player, which is equal to the utility of the middle alternative. It follows that responders are indifferent between accepting and rejecting their middle alternative.

There are other equilibria as well when $c=1 / 2$. Consider for instance the mixed strategy combination following from always proposing the best alternative and accepting the middle alternative with positive probability below one, so $p_{b}=1$ and $0<$ $a_{m}<1$. Every period there is delay with positive probability $1-a_{m}$. Since there is also a positive probability of acceptance, it holds that $\pi_{0}=0$ and $\pi_{1}=\pi_{2}=\pi_{3}=1 / 3$. The expected equilibrium utility is equal to $(1+c+0) / 3=1 / 2=c$. This class of equilibria will be described in Theorem A.4.1. Conditional on being selected as a proposer, a player obtains a utility of $a_{m}+\left(1-a_{m}\right) c>c$. One-shot deviations to proposing the middle alternative or the worst alternative lead to a utility of $c$ and 0 , respectively, so are not profitable. When a player responds to a proposal involving his middle alternative, acceptance leads to a utility of $c$ and rejection leads to a uniform probability distribution on each outcome, leading to an expected utility equal to $c$ as well. It follows that a responder does not have a profitable one-shot deviation.

When $c=1 / 2$, there are also equilibria where players propose their best alternative with probability less than one. Consider the strategy profile induced by $p_{b} \in(0,1)$ and $a_{m}=0$. A proposer randomizes between proposing his best alternative, which is rejected for sure, and proposing his middle alternative, which is surely accepted. Since in each period there is a positive probability that a proposer proposes his best alternative, there is a positive probability of delay in every period. Since there is also a positive probability that a proposer proposes his middle alternative, perpetual delay is 
excluded. We have that $\pi_{0}=0$ and $\pi_{1}=\pi_{2}=\pi_{3}=1 / 3$. The expected equilibrium utility is equal to $c=1 / 2$. This class of equilibria will be described in Theorem A.1. When a proposer proposes his best alternative, it is rejected by both players, and the expected utility for the proposer is equal to $(1+c+0) / 3=c=1 / 2$. When a proposer proposes his middle alternative, it is accepted by the player for whom this is the best alternative, and the utility for the proposer is equal to $c=1 / 2$ as well. It follows that the proposer does not have profitable one-shot deviations. A player responding to a proposal involving his middle alternative obtains utility equal to $(1+c+0) / 3=c$ after a rejection, and utility equal to $c$ after an acceptance, so has no profitable deviation.

In the equilibrium of the previous paragraph, if a player proposes his best alternative, it will be rejected for sure. In utility terms, nothing would change if the proposer would give up his right to propose. The strategy profile induced by $p_{0}>0, p_{b}>0, p_{0}+p_{b}<$ 1 , and $a_{m}=0$ is therefore an equilibrium as well. The strategy profile following from $p_{b} \in(0,1)$ and $a_{m}>0$ is not an equilibrium. If there is a positive probability that a player accepts the middle alternative, then a proposer is strictly better off by proposing his best alternative with probability one.

When $c=1 / 2$, there is also another equilibrium without delay. Consider the strategy profile following from $p_{b}=0$ and $a_{m}=0$. A proposer always proposes his middle alternative, which will be accepted by the player for whom this is the best alternative. As before, it can easily be verified that no player has a profitable one-shot deviation in any subgame. This equilibrium is also described in Theorem A.1.

We finally consider the case $c<1 / 2$. In this case, players receive low utility from their middle alternative, so are less willing to compromise. We will show in Theorem 6.1 that in this case no equilibrium exists, neither symmetric nor asymmetric. For the symmetric equilibria, this is easy to derive. First of all, perpetual disagreement cannot be an equilibrium. Under perpetual disagreement, each player obtains a utility equal to zero. A proposer has a profitable one-shot deviation when proposing his middle alternative, which is going to be accepted by the player for whom this is the best alternative. In any symmetric equilibrium, it should therefore hold that $\pi_{0}=0, \pi_{1}=$ $\pi_{2}=\pi_{3}=1 / 3$, and equilibrium utility is equal to $(1+c+0) / 3>c$. At equilibrium it should then hold that $a_{m}=0$, since an acceptance of the middle alternative gives utility equal to $c$, which is less than rejection with utility $(1+c+0) / 3$. If a proposer proposes his best alternative, it will be rejected, and the proposer receives his continuation utility equal to $(1+c+0) / 3$. This is more than the utility of proposing the middle alternative, which will be accepted, leading to utility equal to $c$. At equilibrium it should therefore hold that $p_{b}=1$. But $p_{b}=1$ and $a_{m}=0$ leads to perpetual disagreement, which we have already argued not to be an equilibrium. Consequently, no symmetric equilibrium exists and, as said before, no asymmetric equilibrium exists either.

\section{Stationary subgame perfect equilibria}

We analyze the extensive-form game of Sect. 2 by examining its stationary subgame perfect equilibria. Suppose a player has to take an action at two subgames that are isomorphic. Then stationarity requires that the player take the same probability mix over actions in both subgames. In defining two subgames to be isomorphic, we follow 
the approach of Maskin and Tirole (2001), which corresponds to the coarsest way of doing so. A subgame perfect equilibrium in stationary strategies is called a stationary subgame perfect equilibrium (SSPE).

Since the continuation game following the selection of a proposer is history independent, we can restrict ourselves to strategies where the proposal is history independent. We denote by $p_{j}^{i}$ the probability that player $i$ proposes $x_{j}$ when he is recognized as proposer. Since the continuation game following a proposal by some player depends only on the proposal made and the identity of the proposer, the rejection probability may only depend on the identity of the proposer and the proposal made, but not on any other aspect of the history. The continuation game starting with the last responder to a proposal depends on the proposal made, but does not depend on the identity of the proposer. We therefore require the response of the last responder to be independent of the identity of the proposer.

The probability that player $i$ rejects a proposal $x_{j}$ by player $h$ is denoted $r_{j h}^{i}$. As explained in the previous paragraph, the notion of a stationary strategy imposes the requirement $r_{32}^{1}=r_{33}^{1}, r_{11}^{2}=r_{13}^{2}$, and $r_{21}^{3}=r_{22}^{3}$. For notational simplicity, we define $r_{0 h}^{i}=1$. We define the set $P$ of admissible proposal probabilities by $P=P^{1} \times P^{2} \times P^{3}$, where

$$
P^{i}=\left\{p^{i} \in \mathbb{R}_{+}^{4} \mid \sum_{j=0,1,2,3} p_{j}^{i}=1\right\}, \quad i=1,2,3
$$

and the set $R$ of admissible rejection probabilities by $R=R^{1} \times R^{2} \times R^{3}$, where

$$
R^{i}=\left\{r^{i} \in[0,1]^{4 \times 2} \mid \text { for } h, h^{\prime} \neq i, r_{0 h}^{i}=1 \text { and } r_{w_{i} h}^{i}=r_{w_{i} h^{\prime}}^{i}\right\}
$$

Given stationary strategies, we can compute the expected utilities of the players. It will be useful to do so conditional on the identity of the proposer. The expected utility of player $i$ conditional on the proposer being player $h$ is denoted by $v_{h}^{i}$. Unconditional expected utility of player $i$ is $z^{i}$ and satisfies $z^{i}=\sum_{h=1}^{3} \rho_{h} v_{h}^{i}$.

Stationarity of the strategies implies that the following recursive system holds,

$$
\begin{aligned}
v_{h}^{i} & =\sum_{j=0}^{3} p_{j}^{h}\left(1-r_{j h}^{h-1} r_{j h}^{h+1}\right) u_{j}^{i}+\sum_{j=0}^{3} p_{j}^{h} r_{j h}^{h-1} r_{j h}^{h+1} z^{i}, \quad i=1,2,3, h=1,2,3 \\
z^{i} & =\sum_{h=1}^{3} \rho_{h} v_{h}^{i}, \quad i=1,2,3 .
\end{aligned}
$$

In the definition of rejection probabilities above, we identify player 0 with player 3 , and player 4 with player 1. Equation (4.1) expresses that the expected utility of player $i$ conditional on the proposer being player $h$ is equal to the sum over all proposals of the probability that player $h$ makes this proposal and that it is accepted by the other 
players times the utility of the proposal plus the probability that player $h$ makes a proposal that is rejected times the continuation utility $z^{i}$.

For the remainder of this section, let $(p, r)$ be an SSPE inducing continuation utilities $v$ and $z$. No player has a profitable deviation at any decision node, so in particular, no player has a profitable one-shot deviation at any decision node. The absence of a profitable one-shot deviation is equivalent to the following set of implications, where in (4.3) it holds that $i \in\{1,2,3\}$ and $j \in\{0,1,2,3\}$,

$$
\begin{aligned}
& p_{j}^{i}>0 \Rightarrow\left(1-r_{j i}^{i-1} r_{j i}^{i+1}\right) u_{j}^{i}+r_{j i}^{i-1} r_{j i}^{i+1} z^{i}=\max _{k \in\{0,1,2,3\}}\left(1-r_{k i}^{i-1} r_{k i}^{i+1}\right) u_{k}^{i}+r_{k i}^{i-1} r_{k i}^{i+1} z^{i}, \\
& r_{j h}^{i}>0 \Rightarrow z^{i} \geq u_{j}^{i} \text { or } r_{j h}^{i^{\prime}}=0, \quad j=1,2,3, h=1,2,3, i=f_{j h}, i^{\prime}=s_{j h}, \\
& r_{j h}^{i}<1 \Rightarrow z^{i} \leq u_{j}^{i} \text { or } r_{j h}^{i^{\prime}}=0, \quad j=1,2,3, h=1,2,3, i=f_{j h}, i^{\prime}=s_{j h}, \\
& r_{j h}^{i}>0 \Rightarrow z^{i} \geq u_{j}^{i}, \quad j=1,2,3, h=1,2,3, i=s_{j h}, \\
& r_{j h}^{i}<1 \Rightarrow z^{i} \leq u_{j}^{i}, \quad j=1,2,3, h=1,2,3, i=s_{j h} .
\end{aligned}
$$

Equality (4.3) expresses that a proposal that is made with positive probability maximizes the sum of instantaneous and continuation utility. We obtain (4.4) by observing that $r_{j h}^{i}>0$ implies $\left(1-r_{j h}^{i^{\prime}}\right) u_{j}^{i}+r_{j h}^{i^{\prime}} z^{i} \geq u_{j}^{i}$; the utility to player $i$ of rejecting proposal $j$ by player $h$ should weakly exceed the utility of acceptance. This inequality is equivalent to $z^{i} \geq u_{j}^{i}$ or $r_{j h}^{i^{\prime}}=0$. The derivation of (4.5)-(4.7) is analogous. Observe that (4.4)-(4.5) correspond to the cases where player $i$ is the first voter to accept or reject a proposal, and (4.6)-(4.7) to the cases where player $i$ is the second voter to make such a decision.

We now derive several properties of SSPEs, thereby reducing (4.3)-(4.7) to a considerably simpler system. The first property states that forever delay with probability 1 is not an SSPE. Indeed, forever delay with probability 1 implies, for every $i, z^{i}=0$ and $v^{i}=0$. By (4.3), player 1 should obtain expected utility 0 from proposing $x_{1}$, which can only be the case if $r_{11}^{2}=r_{11}^{3}=1$. By (4.4), $r_{11}^{3}=1$ implies $z^{3} \geq u_{1}^{3}$ or $r_{11}^{2}=0$. This leads to a contradiction as $z^{3}=0<u_{1}^{3}$ and $r_{11}^{2}=1$. It follows that forever delay with probability 1 is not an SSPE.

We have derived that some player makes with positive probability a proposal that is accepted with positive probability. Since such a player is recognized with positive probability, the probability that negotiations have not terminated at period $t$ goes to zero as $t$ goes to infinity.

Theorem 4.1 It holds that $\pi_{0}=0$, every SSPE leads with probability 1 to agreement in finite time.

Since $\pi_{0}=0$, each $z^{i}$ is therefore a weighted average of $u_{j}^{i}, j=1,2,3$, with $\pi_{j} \in[0,1]$ such that $\pi_{1}+\pi_{2}+\pi_{3}=1$ independent of $i$. It holds in particular that $\pi_{j}>0$ for some $j=1,2,3$ and $\left(z^{1}, z^{2}, z^{3}\right) \neq 0$.

Theorem 4.1 shows that bargaining under exogenous recognition probabilities is a road map to overcome the Condorcet paradox. Given the indeterminacy of many cooperative theories about the Condorcet paradox, this result already suggests a great potential in further elaborating the bargaining approach. 
Conditions (4.1)-(4.7) are necessary conditions for an SSPE. For games with discounting, these necessary conditions are also sufficient. However, in the absence of discounting, the system (4.1)-(4.7) is degenerate when for every proposer $h=1,2,3$ it holds that $\sum_{j=0}^{3} p_{j}^{h} r_{j h}^{h-1} r_{j h}^{h+1}=1$, i.e. the case of perpetual delay, and admits solutions for conditional utilities $v_{h}^{i}$ and continuation utilities $z^{i}$ that are not the actual utilities of perpetual delay, which are equal to zero. Since perpetual delay is not an equilibrium and the system (4.1)-(4.7) is not degenerate when for some $h=1,2,3$ it holds that $\sum_{j=0}^{3} p_{j}^{h} r_{j h}^{h-1} r_{j h}^{h+1}<1$, we obtain the necessary and sufficient conditions as specified in Theorem 4.2. This approach turns out to apply for quite general bargaining models as has been demonstrated in Herings and Houba (2015).

Theorem 4.2 The strategy profile $(p, r) \in P \times R$ is an SSPE if and only if there is $h$ such that $\sum_{j=0}^{3} p_{j}^{h} r_{j h}^{h-1} r_{j h}^{h+1}<1$ and there is $v \in \mathbb{R}^{3 \times 3}$ and $z \in \mathbb{R}^{3}$ such that (4.1)-(4.7) hold.

In the next step, we use the characterization of SSPE given in Theorem 4.2 to derive a number of intuitive properties that equilibria should satisfy.

Theorem 4.3 Let the strategy profile $(p, r)$ be an SSPE with continuation utilities $z$ and outcome probability distribution $\pi$. Then

$$
\begin{aligned}
& p_{w_{i}}^{i}=0, \quad i=1,2,3, \\
& r_{w_{i} h}^{i}=1, \quad i=1,2,3, h \neq i, \\
& r_{21}^{2}=r_{32}^{3}=r_{13}^{1}=0, \\
& r_{31}^{2} r_{31}^{3}=r_{12}^{1} r_{12}^{3}=r_{23}^{1} r_{23}^{2}=0, \\
& z^{i}>u_{w_{i}}^{i}, \quad i=1,2,3, \\
& z^{i}<u_{b_{i}}^{i}, \quad i=1,2,3, \\
& \pi_{1}, \pi_{2}, \pi_{3}>0 .
\end{aligned}
$$

According to (4.12), each player $i$ has $z^{i}$ strictly exceeding the utility $u_{w_{i}}^{i}$ of his worst alternative and, according to (4.13), has $z^{i}$ strictly lower than the utility of his best alternative, $u_{b_{i}}^{i}$. It then follows that any voter rejects his worst alternative for sure as expressed in (4.9). It follows from (4.10) that a proposal where a player proposes his middle alternative is accepted by the player for whom this is the best alternative, whereas (4.11) claims that proposing the worst alternative leads to an acceptance. The recognized player can therefore always conclude the bargaining for sure by proposing his worst or his middle alternative. As a corollary, a recognized player will never propose his worst alternative, because he can do strictly better by proposing his middle alternative, and (4.8) follows. Finally, (4.14) states that, ex ante, every alternative is accepted with strictly positive probability.

The next result claims that there is no loss of generality in restricting the analysis to proposer-independent rejection probabilities.

Theorem 4.4 If $(p, r) \in P \times R$ is an SSPE inducing utilities $v$ and $z$, then there is also an $\operatorname{SSPE}(p, \bar{r}) \in P \times R$ inducing utilities $v$ and $z$ such that $\bar{r}$ is proposer- 
independent, i.e. $\bar{r}_{j h}^{i}=\bar{r}_{j h^{\prime}}^{i}$ for all $i, j, h$, and $h^{\prime}$. Moreover, $\bar{r}$ can be defined by setting, for $i=1,2,3, \bar{r}_{b_{i} i+1}^{i}=0, \bar{r}_{m_{i} i-1}^{i}=r_{m_{i} i+1}^{i}$, and $\bar{r}_{j h}^{i}=r_{j h}^{i}$, otherwise.

By virtue of Theorem 4.4, we may drop the subscript indicating the proposer from the notation of a rejection probability. It is also more convenient now to express all equations in terms of acceptance probabilities rather than rejection probabilities. The set of proposer-independent acceptance probabilities is $A=A^{1} \times A^{2} \times A^{3}$, where

$$
A^{i}=\left\{a^{i} \in[0,1]^{4} \mid a_{0}^{i}=0\right\} .
$$

It follows from Theorem 4.3 that at an $\operatorname{SSPE}(p, \bar{a}) \in P \times A$, for every player $i$, $p_{w_{i}}^{i}=0, \bar{a}_{w_{i}}^{i}=0$, and $\bar{a}_{b_{i}}^{i}=1$. The only variables that have not yet been determined are $p_{0}^{i}, p_{m_{i}}^{i}, p_{b_{i}}^{i}$, and $\bar{a}_{m_{i}}^{i}$. It seems intuitive that the recognized player is better off making some proposal instead of not making a proposal, so $p_{0}^{i}$ should be equal to 0 . As we will show in Appendix A.1, for some parameter values we can have $p_{0}^{i}>0$ for some $i$. In such cases, however, there also exists an $\operatorname{SSPE}(\bar{p}, \bar{a}) \in P \times A$ with $\bar{p}_{0}^{i}=0$ for every player $i$ that yields exactly the same utilities for everyone. This implies that in characterizing the set of SSPEs, we may first search for SSPEs $(\bar{p}, \bar{a}) \in P \times A$ with $\bar{p}_{0}^{i}=0$ for every player $i$. Indeed, if $(p, \bar{a})$ is an SSPE with $p_{0}^{i} \neq 0$ for some $i$, then $(\bar{p}, \bar{a})$ is also an SSPE, where $\bar{p}_{b_{i}}^{i}=p_{b_{i}}^{i}+p_{0}^{i}, \bar{p}_{0}^{i}=0$, and $\bar{p}_{j}^{i}=p_{j}^{i}$ for $j \neq 0, b_{i}$. By the definition of SSPE it should not be profitable to propose $x_{b_{i}}$ instead of $x_{0}$. This means that either $x_{b_{i}}$ is rejected with probability 1 when proposed or $z^{i}=u_{b_{i}}^{i}$. The latter case contradicts (4.13), so we only have to consider the former case. Since we are considering SSPEs, the change in strategy from not making a proposal to proposing one's best alternative, which is rejected with probability 1 , is not affecting the payoffs of anyone, and is also an SSPE.

Theorem 4.5 gives an easy characterization of SSPEs $(\bar{p}, \bar{a}) \in P \times A$ where no player gives up the right to make a proposal, i.e. $\bar{p}_{0}^{i}=0$ for every player $i$.

Theorem 4.5 The strategy profile $(\bar{p}, \bar{a}) \in P \times A$ is an SSPE where all players make a proposal with probability one if and only if for $i=1,2,3, \bar{p}_{0}^{i}=\bar{p}_{w_{i}}^{i}=0$, $\bar{a}_{b_{i}}^{i}=1, \bar{a}_{w_{i}}^{i}=0$, and there is $\bar{\pi} \in \mathbb{R}_{++}^{3}$ and $\bar{z} \in \mathbb{R}^{3}$ such that

$$
\begin{aligned}
& \bar{p}_{m_{i}}^{i}>0 \Rightarrow u_{m_{i}}^{i} \geq \bar{a}_{m_{i-1}}^{i-1} u_{b_{i}}^{i}+\left(1-\bar{a}_{m_{i-1}}^{i-1}\right) \bar{z}^{i}, \quad i=1,2,3, \\
& \bar{p}_{b_{i}}^{i}>0 \Rightarrow \bar{a}_{m_{i-1}}^{i-1} u_{b_{i}}^{i}+\left(1-\bar{a}_{m_{i-1}}^{i-1}\right) \bar{z}^{i} \geq u_{m_{i}}^{i}, \quad i=1,2,3, \\
& \bar{a}_{m_{i}}^{i}<1 \Rightarrow \bar{z}^{i} \geq u_{m_{i}}^{i}, \quad i=1,2,3, \\
& \bar{a}_{m_{i}}^{i}>0 \Rightarrow \bar{z}^{i} \leq u_{m_{i}}^{i}, \quad i=1,2,3, \\
& \bar{\pi}_{1} u_{1}^{i}+\bar{\pi}_{2} u_{2}^{i}+\bar{\pi}_{3} u_{3}^{i}=\bar{z}^{i}, \quad i=1,2,3, \\
& \bar{\pi}_{1}+\bar{\pi}_{2}+\bar{\pi}_{3}=1, \\
& \bar{\pi}_{1}: \bar{\pi}_{2}=\rho_{1} \bar{p}_{b_{1}}^{1} \bar{a}_{1}^{3}+\rho_{3} \bar{p}_{m_{3}}^{3}: \rho_{2} \bar{p}_{b_{2}}^{2} \bar{a}_{2}^{1}+\rho_{1} \bar{p}_{m_{1}}^{1}, \\
& \bar{\pi}_{2}: \bar{\pi}_{3}=\rho_{2} \bar{p}_{b_{2}}^{2} \bar{a}_{2}^{1}+\rho_{1} \bar{p}_{m_{1}}^{1}: \rho_{3} \bar{p}_{b_{3}}^{3} \bar{a}_{3}^{2}+\rho_{2} \bar{p}_{m_{2}}^{2} .
\end{aligned}
$$




\section{Equilibrium types}

The results of the previous section show that player $i$ faces two dilemmas. First, by what probability $p_{m_{i}}^{i}$ will I propose my middle alternative $x_{m_{i}}$ knowing it will be accepted for sure instead of taking the risk involved in proposing my best alternative. Second, by what probability $a_{m_{i}}^{i}$ will I accept my middle alternative $x_{m_{i}}$ when offered to me knowing that rejecting it leads to a gamble over my top three alternatives including my worst. These dilemmas concern the SSPE values of $p_{m_{i}}^{i}$ and $a_{m_{i}}^{i}$ that also pin down $p_{b_{i}}^{i}=1-p_{m_{i}}^{i}$.

The answer to the first dilemma results in four possible types of equilibrium. The first one is where every player $i$ has a positive $\bar{p}_{m_{i}}^{i}$. This case is analyzed in Appendix A.1. The other types of equilibria are characterized by two, one, and none of the players having a positive $\bar{p}_{m_{i}}^{i}$ and are treated in Appendix A.2, A.3, and A.4, respectively. The answer to the second dilemma is intimately related to the value of the equilibrium continuation utility $\bar{z}^{i}$. We will show that all SSPEs have the property that $\bar{z}^{i} \leq u_{m_{i}}^{i}$. Then it follows that $\bar{a}_{m_{i}}^{i}=1$ if $\bar{z}^{i}<u_{m_{i}}^{i}$, whereas values for $\bar{a}_{m_{i}}^{i}$ strictly below 1 are admitted when $\bar{z}^{i}=u_{m_{i}}^{i}$.

Theorem A.1 collects the necessary and sufficient conditions under which an SSPE with three players having a positive $\bar{p}_{m_{i}}^{i}$ exists. These conditions hold in degenerate cases only. Moreover, in any such SSPE it holds that $\bar{z}^{i}=u_{m_{i}}^{i}$ for every player $i$. We show that SSPEs with two players having a positive $\bar{p}_{m_{i}}^{i}$ do not exist. For the case with one of the players having a positive $\bar{p}_{m_{i}}^{i}$ there exist two equilibrium subtypes, depending on the number of players with $\bar{z}^{i}<u_{m_{i}}^{i}$. These subtypes are treated in the Subsects. A.3.1, when there is one such player, and A.3.2, when there are two such players. Theorems A.3.1 and A.3.2 provide necessary and sufficient conditions under which such SSPEs exist. For the case with none of the players having a positive $\bar{p}_{m_{i}}^{i}$ there exist four equilibrium subtypes, again depending on the number of players with $\bar{z}^{i}=u_{m_{i}}^{i}$. These subtypes are treated in the Subsects. A.4.1, A.4.2, A.4.3, and A.4.4 of Appendix A.4, where Subsect. A.4.k treats the case when there are $k-1$ players with $\bar{z}^{i}<u_{m_{i}}^{i}$. Theorems A.4.1, A.4.2, A.4.3, and A.4.4 collect the necessary and sufficient conditions under which such SSPEs exist.

Table 2 summarizes the characteristics of the SSPEs as found in the Appendix. The equilibrium types and subtypes lead to a total of seven cases, with three cases being degenerate. The cases A.3.1, A.3.2, A.4.2, and A.4.3 have three rows, corresponding to permutations of the players' roles. Four cases, A.3.1, A.3.2, A.4.2, and A.4.4, are robust in the sense of having positive Lebesgue measure in the parameter space. Case A.4.4 corresponds to an SSPE in pure strategies.

Table 3 shows the necessary and sufficient conditions for which particular types of equilibria exist. To explain these conditions, it is instructive to define the risk coefficient $\alpha_{i}$ of player $i$ by 
Table 2 Characteristics of the various types of equilibrium

\begin{tabular}{|c|c|c|c|}
\hline Theorem & Proposals & Utilities & Occurrence \\
\hline A. 1 & $\bar{p}_{2}^{1}>0 \quad \bar{p}_{3}^{2}>0 \quad \bar{p}_{1}^{3}>0$ & $\bar{z}^{1}=u_{2}^{1} \quad \bar{z}^{2}=u_{3}^{2} \quad \bar{z}^{3}=u_{1}^{3}$ & Degenerate \\
\hline \multirow[t]{3}{*}{ A.3.1 } & $\bar{p}_{2}^{1}=0 \quad \bar{p}_{3}^{2}=0 \quad \bar{p}_{1}^{3}>0$ & $\bar{z}^{1}=u_{2}^{1} \quad \bar{z}^{2}=u_{3}^{2} \quad \bar{z}^{3}<u_{1}^{3}$ & \\
\hline & $\bar{p}_{2}^{1}=0 \quad \bar{p}_{3}^{2}>0 \quad \bar{p}_{1}^{3}=0$ & $\bar{z}^{1}=u_{2}^{1} \quad \bar{z}^{2}<u_{3}^{2} \quad \bar{z}^{3}=u_{1}^{3}$ & \\
\hline & $\bar{p}_{2}^{1}>0 \quad \bar{p}_{3}^{2}=0 \quad \bar{p}_{1}^{3}=0$ & $\bar{z}^{1}<u_{2}^{1} \quad \bar{z}^{2}=u_{3}^{2} \quad \bar{z}^{3}=u_{1}^{3}$ & \\
\hline \multirow[t]{3}{*}{ A.3.2 } & $\bar{p}_{2}^{1}=0 \quad \bar{p}_{3}^{2}=0 \quad \bar{p}_{1}^{3}>0$ & $\bar{z}^{1}<u_{2}^{1} \quad \bar{z}^{2}=u_{3}^{2} \quad \bar{z}^{3}<u_{1}^{3}$ & \\
\hline & $\bar{p}_{2}^{1}=0 \quad \bar{p}_{3}^{2}>0 \quad \bar{p}_{1}^{3}=0$ & $\bar{z}^{1}=u_{2}^{1} \quad \bar{z}^{2}<u_{3}^{2} \quad \bar{z}^{3}<u_{1}^{3}$ & \\
\hline & $\bar{p}_{2}^{1}>0 \quad \bar{p}_{3}^{2}=0 \quad \bar{p}_{1}^{3}=0$ & $\bar{z}^{1}<u_{2}^{1} \quad \bar{z}^{2}<u_{3}^{2} \quad \bar{z}^{3}=u_{1}^{3}$ & \\
\hline A. 4.1 & $\bar{p}_{2}^{1}=0 \quad \bar{p}_{3}^{2}=0 \quad \bar{p}_{1}^{3}=0$ & $\bar{z}^{1}=u_{2}^{1} \quad \bar{z}^{2}=u_{3}^{2} \quad \bar{z}^{3}=u_{1}^{3}$ & Degenerate \\
\hline \multirow[t]{3}{*}{ A. 4.2} & $\bar{p}_{2}^{1}=0 \quad \bar{p}_{3}^{2}=0 \quad \bar{p}_{1}^{3}=0$ & $\bar{z}^{1}=u_{2}^{1} \quad \bar{z}^{2}=u_{3}^{2} \quad \bar{z}^{3}<u_{1}^{3}$ & \\
\hline & $\bar{p}_{2}^{1}=0 \quad \bar{p}_{3}^{2}=0 \quad \bar{p}_{1}^{3}=0$ & $\bar{z}^{1}=u_{2}^{1} \quad \bar{z}^{2}<u_{3}^{2} \quad \bar{z}^{3}=u_{1}^{3}$ & \\
\hline & $\bar{p}_{2}^{1}=0 \quad \bar{p}_{3}^{2}=0 \quad \bar{p}_{1}^{3}=0$ & $\bar{z}^{1}<u_{2}^{1} \quad \bar{z}^{2}=u_{3}^{2} \quad \bar{z}^{3}=u_{1}^{3}$ & \\
\hline \multirow[t]{3}{*}{ A. 4.3} & $\bar{p}_{2}^{1}=0 \quad \bar{p}_{3}^{2}=0 \quad \bar{p}_{1}^{3}=0$ & $\bar{z}^{1}=u_{2}^{1} \quad \bar{z}^{2}<u_{3}^{2} \quad \bar{z}^{3}<u_{1}^{3}$ & Degenerate \\
\hline & $\bar{p}_{2}^{1}=0 \quad \bar{p}_{3}^{2}=0 \quad \bar{p}_{1}^{3}=0$ & $\bar{z}^{1}<u_{2}^{1} \quad \bar{z}^{2}=u_{3}^{2} \quad \bar{z}^{3}<u_{1}^{3}$ & Degenerate \\
\hline & $\bar{p}_{2}^{1}=0 \quad \bar{p}_{3}^{2}=0 \quad \bar{p}_{1}^{3}=0$ & $\bar{z}^{1}<u_{2}^{1} \quad \bar{z}^{2}<u_{3}^{2} \quad \bar{z}^{3}=u_{1}^{3}$ & Degenerate \\
\hline A.4.4 & $\bar{p}_{2}^{1}=0 \quad \bar{p}_{3}^{2}=0 \quad \bar{p}_{1}^{3}=0$ & $\bar{z}^{1}<u_{2}^{1} \quad \bar{z}^{2}<u_{3}^{2} \quad \bar{z}^{3}<u_{1}^{3}$ & \\
\hline
\end{tabular}

Table 3 Conditions under which various types of equilibrium exist

\begin{tabular}{|c|c|c|c|c|c|}
\hline \multirow{2}{*}{$\begin{array}{l}\text { Theorem } \\
\text { A.1 }\end{array}$} & \multirow{2}{*}{$\begin{array}{l}\text { Conditions on } \alpha \\
\alpha_{1} \alpha_{2} \alpha_{3}=1\end{array}$} & \multicolumn{3}{|c|}{ Conditions on $\rho$} & \multirow{2}{*}{$\frac{\text { Occurrence }}{\text { Degenerate }}$} \\
\hline & & & & & \\
\hline \multirow[t]{3}{*}{ A. 3.1} & $\alpha_{1} \alpha_{2} \alpha_{3}<1$ & $\frac{\rho_{1}}{\rho_{3}}<\beta_{1}$ & & $\rho_{2} \geq \alpha_{3} \beta_{3}$ & \\
\hline & & $\frac{\rho_{3}}{\rho_{2}}<\beta_{3}$ & & $\rho_{1} \geq \alpha_{2} \beta_{2}$ & \\
\hline & & $\frac{\rho_{2}}{\rho_{1}}<\beta_{2}$ & & $\rho_{3} \geq \alpha_{1} \beta_{1}$ & \\
\hline \multirow[t]{3}{*}{ A. 3.2} & $\alpha_{1} \alpha_{2} \alpha_{3}<1$ & $\frac{\rho_{1}}{\rho_{2}}<\alpha_{2}$ & & $\rho_{2}<\alpha_{3} \beta_{3}$ & \\
\hline & & $\frac{\rho_{3}}{\rho_{1}}<\alpha_{1}$ & & $\rho_{1}<\alpha_{2} \beta_{2}$ & \\
\hline & & $\frac{\rho_{2}}{\rho_{3}}<\alpha_{3}$ & & $\rho_{3}<\alpha_{1} \beta_{1}$ & \\
\hline A. 4.1 & $\alpha_{1} \alpha_{2} \alpha_{3}=1$ & & & & Degenerate \\
\hline \multirow[t]{3}{*}{ A. 4.2} & $\alpha_{1} \alpha_{2} \alpha_{3}<1$ & $\frac{\rho_{1}}{\rho_{3}} \geq \beta_{1}$ & $\frac{\rho_{1}}{\rho_{2}} \leq \alpha_{2}$ & $\frac{\rho_{3}}{\rho_{1}} \geq \alpha_{1}$ & \\
\hline & & $\frac{\rho_{3}}{\rho_{2}} \geq \beta_{3}$ & $\frac{\rho_{3}}{\rho_{1}} \leq \alpha_{1}$ & $\frac{\rho_{2}}{\rho_{3}} \geq \alpha_{3}$ & \\
\hline & & $\frac{\rho_{2}}{\rho_{1}} \geq \beta_{2}$ & $\frac{\rho_{2}}{\rho_{3}} \leq \alpha_{3}$ & $\frac{\rho_{1}}{\rho_{2}} \geq \alpha_{2}$ & \\
\hline \multirow[t]{3}{*}{ A. 4.3} & $\alpha_{1} \alpha_{2} \alpha_{3}<1$ & $\frac{\rho_{3}}{\rho_{1}}=\alpha_{1}$ & & $\rho_{1}<\frac{1}{1+\alpha_{1}+\alpha_{1} \alpha_{3}}$ & Degenerate \\
\hline & & $\frac{\rho_{1}}{\rho_{2}}=\alpha_{2}$ & & $\rho_{2}<\frac{1}{1+\alpha_{2}+\alpha_{1} \alpha_{2}}$ & Degenerate \\
\hline & & $\frac{\rho_{2}}{\rho_{3}}=\alpha_{3}$ & & $\rho_{3}<\frac{1}{1+\alpha_{3}+\alpha_{2} \alpha_{3}}$ & Degenerate \\
\hline A. 4.4 & $\alpha_{1} \alpha_{2} \alpha_{3}<1$ & $\frac{\rho_{1}}{\rho_{2}}>\alpha_{2}$ & $\frac{\rho_{2}}{\rho_{3}}>\alpha_{3}$ & $\frac{\rho_{3}}{\rho_{1}}>\alpha_{1}$ & \\
\hline
\end{tabular}




$$
\alpha_{i}=\frac{u_{b_{i}}^{i}-u_{m_{i}}^{i}}{u_{m_{i}}^{i}-u_{w_{i}}^{i}}, \quad i=1,2,3 .
$$

Moreover, for notational convenience, we define

$$
\begin{aligned}
& \beta_{1}=\frac{1-\alpha_{1} \alpha_{2} \alpha_{3}}{\alpha_{1}+\alpha_{1} \alpha_{3}+\alpha_{1} \alpha_{2} \alpha_{3}}, \quad \beta_{2}=\frac{1-\alpha_{1} \alpha_{2} \alpha_{3}}{\alpha_{2}+\alpha_{1} \alpha_{2}+\alpha_{1} \alpha_{2} \alpha_{3}}, \quad \text { and } \\
& \beta_{3}=\frac{1-\alpha_{1} \alpha_{2} \alpha_{3}}{\alpha_{3}+\alpha_{2} \alpha_{3}+\alpha_{1} \alpha_{2} \alpha_{3}} .
\end{aligned}
$$

Table 3 demonstrates that the necessary and sufficient conditions for SSPE existence can be formulated in terms of the players' risk coefficients (since also $\beta_{i}$ can be expressed in terms of $\alpha_{1}, \alpha_{2}$, and $\alpha_{3}$ ) and the recognition probability vector $\rho$ only.

The risk coefficient is closely related to the concept of risk limit as introduced in Zeuthen (1930) and further developed in Harsanyi (1977). The risk limit is defined in a setting with two players and three outcomes. There is the outcome proposed by the player himself, say $y_{1}$, the outcome proposed by his opponent, say $y_{2}$, and the disagreement outcome, say $y_{0}$. The risk limit of a player is then defined as the probability on the disagreement outcome for which he would be indifferent between getting the disagreement outcome with that probability and $y_{1}$ with the remaining probability, and getting outcome $y_{2}$ for sure. In a formula the risk limit $\ell$ is given by

$$
\ell=\frac{u\left(y_{1}\right)-u\left(y_{2}\right)}{u\left(y_{1}\right)-u\left(y_{0}\right)} .
$$

This paper involves three players and four alternatives (we now count the disagreement outcome as one alternative), so the risk limit is not directly applicable. However, if we define $y_{1}$ as the best alternative $x_{b_{i}}$ for player $i, y_{2}$ as his middle alternative $x_{m_{i}}$, and $y_{0}$ as his worst alternative $x_{w_{i}}$, then a straightforward calculation reveals that

$$
\ell_{i}=\frac{\alpha_{i}}{1+\alpha_{i}}
$$

Alternatively, we can write $\alpha_{i}=\ell_{i} /\left(1-\ell_{i}\right)$.

A player $i$ who is indifferent between getting $x_{m_{i}}$ for sure and a fair lottery on $x_{b_{i}}$ and $x_{w_{i}}$ has a risk coefficient of 1 . A player with a risk coefficient above 1 prefers the lottery, a player with a risk coefficient below 1 prefers getting his middle alternative for sure. It is immediate from Table 3 that a necessary condition for SSPE existence is $\alpha_{1} \alpha_{2} \alpha_{3} \leq 1$, or equivalently, $\sqrt[3]{\alpha_{1} \alpha_{2} \alpha_{3}} \leq 1$. In words this condition expresses that the geometric mean of the players' risk coefficients is less than or equal to 1 . In the next section this condition is also shown to be sufficient for SSPE existence.

The robust cases have the following defining characteristics: First, conditional on being recognized, at most one player randomizes between his best and middle alternative, and the other players always propose their best alternative for sure. To put it differently, at most one player proposes cautiously and the others aggressively. Second, the number of players who propose their best alternative and get it accepted for 
sure can be any number ranging from one to three, but it cannot be zero. Third, at the start of any bargaining round during ongoing negotiations, all players can realize, in expectation, an SSPE utility that is at most the utility of the middle alternative, so $\bar{z}^{i} \leq u_{m_{i}}^{i}$. In case the inequality is strict, player $i$ accepts his middle alternative for sure, whenever it is on the table. In any SSPE, this will provoke player $i+1$, for whom $m_{i}$ is the best alternative, to propose aggressively whenever he is recognized. Fourth, conditional on being recognized, a player realizes a utility weakly exceeding the utility of his middle alternative, so $\bar{v}_{i}^{i} \geq u_{m_{i}}^{i}$. Moreover, it can be shown that there is a strict advantage in being recognized, so $\bar{v}_{i}^{i}>\bar{z}^{i}$.

\section{Existence and uniqueness of SSPE}

Table 3 shows that there are seven different types of SSPE and presents the necessary and sufficient conditions for the existence of each type in terms of six parameters: $\alpha_{1}, \alpha_{2}, \alpha_{3}, \rho_{1}, \rho_{2}$, and $\rho_{3}$. Theorem 6.1 is about the necessary and sufficient conditions on the parameters for the existence of any of the seven types of SSPE, so corresponds to the union of the seven sets of parameter values of Table 3. Surprisingly, this leads to the very simple necessary and sufficient condition that the geometric mean of the risk coefficients be less than or equal to $1: \sqrt[3]{\alpha_{1} \alpha_{2} \alpha_{3}} \leq 1$ or, equivalently $\alpha_{1} \alpha_{2} \alpha_{3} \leq 1$.

Theorem 6.1 There exists an SSPE if and only if $\alpha_{1} \alpha_{2} \alpha_{3} \leq 1$.

The necessary and sufficient condition for SSPE existence requires risk coefficients to be sufficiently low on average. It allows for one or two risk coefficients that are larger than one, but then at least one player's risk coefficient should be sufficiently below one. A player with a low risk coefficient prefers his middle alternative over a lottery involving his worst and best alternative, and is therefore more inclined to accept proposals offering his middle alternative. The uncertainty over outcomes resulting from the rejection of a proposal helps to avoid the Condorcet paradox and leads to equilibrium existence.

What can be said when $\alpha_{1} \alpha_{2} \alpha_{3}>1$ ? An SSPE does not exist by Theorem 6.1. Nevertheless, it is conceivable that weaker versions of equilibrium, where stationarity and perfection requirements are no longer imposed, do exist. Suppose that we change the utilities in the game in the following way. Whenever an agreement is reached, players receive the payoff related to this agreement in every period following the agreement and the utility of a player is determined by the average reward criterion. The resulting game thereby falls into the class of average reward stochastic games. Since the game also belongs to the subclass of three-player absorbing games, it follows from Solan (1999) that an $\varepsilon$-equilibrium payoff exists for every $\varepsilon>0$. Since our game also belongs to the class of perfect information stochastic games, the existence of a Nash equilibrium follows from the results of Thuijsman and Raghavan (1997). Finally, our game is also a recursive perfect information game with non-negative payoffs, a class for which Flesch et al. (2010) demonstrate the existence of a subgame-perfect $\varepsilon$-equilibrium for every $\varepsilon>0$.

In our model, SSPE utilities may not be unique and there might be infinitely many SSPE utilities. This occurs under the conditions of Theorem A.4.3. The following 
result demonstrates that such examples are degenerate in the sense that this set of games has a closure with Lebesgue measure zero. To compute the Lebesgue measure of a set of games, we consider a game $(u, \rho)$ as an element of $\mathbb{R}^{9} \times \mathbb{R}^{2}$, where we identify $\rho$ by its first two coordinates. To require the zero Lebesgue measure property for the closure of a set of games evidently implies this property for the set of games itself, but not vice versa, as for instance illustrated by the set of rational numbers.

Theorem 6.2 Consider the set of games $(u, \rho) \in \mathcal{G}$ such that $\alpha_{1} \alpha_{2} \alpha_{3} \leq 1$. Except for a subset of games whose closure has Lebesgue measure zero, SSPE utilities are unique and there is a unique SSPE with proposer-independent acceptance probabilities.

The generic uniqueness of SSPEs with proposer-independent acceptance probabilities enables us to carry out meaningful comparative statics exercises, the subject of the next two sections.

\section{Delay and cycles}

We analyze the extent to which there can be delay in an SSPE. If the probability of delay in a single bargaining round is $\delta$, then the expected delay is equal to $\delta /(1-\delta)$ periods. Using the results of the Appendix, it is a straightforward exercise to compute the probability of delay in a single bargaining round. Table 4 gives an overview of the delay probabilities. In the case of Theorems A.1 and A.4.3, the delay probability is given by an interval. This means that for every value of delay in the interval, there is an SSPE with that probability of delay.

An important question is whether there always exists some vector of recognition probabilities $\rho$ such that the corresponding SSPE does not involve delay.

Theorem 7.1 Let $u$ be such that $\alpha_{1} \alpha_{2} \alpha_{3} \leq 1$. Then there is a $\rho$ such that the game $(u, \rho)$ has an SSPE without delay.

If we think of the parameters $\rho_{i}$ as a measure of bargaining power, then Theorem 7.1 makes clear that irrespective of the players' utility functions, delay in bargaining can be avoided under an appropriate distribution of bargaining power.

The intuition behind Theorem 7.1 is the following. Suppose that every player proposes his best alternative with probability 1 , meaning that alternatives are implemented according to the probability vector $\rho$. Under the condition $\alpha_{1} \alpha_{2} \alpha_{3} \leq 1$ it is always possible to choose $\rho$ in such a way that every player $i$ 's continuation payoff is less than or equal to $u_{m_{i}}^{i}$. In particular, this means that players with high risk coefficients should have low recognition probabilities. Given a continuation payoff below $u_{m_{i}}^{i}$, player $i$ accepts alternative $x_{m_{i}}$ with probability 1, which in turn makes it optimal for every player to propose his best alternative with probability 1 .

The next result shows that the expected delay in bargaining goes to infinity when one player has almost all the bargaining power. We model this by taking a sequence of recognition probability vectors that converges to a unit vector and show that the limit of the SSPE delay probability is equal to 1 . 
Table 4 Delay probabilities

\begin{tabular}{|c|c|c|}
\hline Theorem & Delay probability & Occurrence \\
\hline A. 1 & {$\left[1-\left(1+\alpha_{2}+\alpha_{1} \alpha_{2}\right) \min \left\{\rho_{1}, \frac{\rho_{2}}{\alpha_{1} \alpha_{2}}, \frac{\rho_{3}}{\alpha_{2}}\right\}, 1\right)$} & Degenerate \\
\hline \multirow[t]{3}{*}{ A.3.1 } & $\rho_{2}+\left(\alpha_{1} \alpha_{2} \alpha_{3}\left(1+\beta_{2}\right)-\beta_{2}\right)\left(1-\rho_{2}\right)$ & \\
\hline & $\rho_{1}+\left(\alpha_{1} \alpha_{2} \alpha_{3}\left(1+\beta_{1}\right)-\beta_{1}\right)\left(1-\rho_{1}\right)$ & \\
\hline & $\rho_{3}+\left(\alpha_{1} \alpha_{2} \alpha_{3}\left(1+\beta_{3}\right)-\beta_{3}\right)\left(1-\rho_{3}\right)$ & \\
\hline \multirow[t]{3}{*}{ A.3.2 } & $\frac{\alpha_{3}-\left(1+\alpha_{2}\right) \alpha_{3} \rho_{2}}{\alpha_{3}+\rho_{2}}$ & \\
\hline & $\frac{\alpha_{2}-\left(1+\alpha_{1}\right) \alpha_{2} \rho_{1}}{\alpha_{2}+\rho_{1}}$ & \\
\hline & $\frac{\alpha_{1}-\left(1+\alpha_{3}\right) \alpha_{1} \rho_{3}}{\alpha_{1}+\rho_{3}}$ & \\
\hline A. 4.1 & $1-\left(1+\alpha_{2}+\alpha_{1} \alpha_{2}\right) \min \left\{\frac{\rho_{1}}{\alpha_{2}}, \rho_{2}, \frac{\rho_{3}}{\alpha_{1} \alpha_{2}}\right\}$ & Degenerate \\
\hline \multirow[t]{3}{*}{ A. 4.2} & $1-\frac{1+\alpha_{2}+\alpha_{1} \alpha_{2}}{\alpha_{2}} \rho_{1}$ & \\
\hline & $1-\frac{1+\alpha_{1}+\alpha_{1} \alpha_{3}}{\alpha_{1}} \rho_{3}$ & \\
\hline & $1-\frac{1+\alpha_{3}+\alpha_{2} \alpha_{3}}{\alpha_{3}} \rho_{2}$ & \\
\hline \multirow[t]{3}{*}{ A. 4.3} & $D_{1}$ & Degenerate \\
\hline & $D_{2}$ & Degenerate \\
\hline & $D_{3}$ & Degenerate \\
\hline A. 4.4 & 0 & \\
\hline \multicolumn{3}{|c|}{$D_{1}=\left[0,1-\left(1+\alpha_{1}+\alpha_{1} \alpha_{3}\right) \rho_{1}\right) \cap\left(1-\frac{\left(1+\alpha_{2}+\alpha_{1} \alpha_{2}\right) \rho_{1}}{\alpha_{2}}, 1-\frac{\left(1+\alpha_{2}+\alpha_{1} \alpha_{2}\right) \rho_{1}}{\alpha_{2}+\rho_{1}}\right]$} \\
\hline \multicolumn{3}{|c|}{$D_{2}=\left[0,1-\left(1+\alpha_{2}+\alpha_{1} \alpha_{2}\right) \rho_{2}\right) \cap\left(1-\frac{\left(1+\alpha_{3}+\alpha_{2} \alpha_{3}\right) \rho_{2}}{\alpha_{3}}, 1-\frac{\left(1+\alpha_{3}+\alpha_{2} \alpha_{3}\right) \rho_{2}}{\alpha_{3}+\rho_{2}}\right]$} \\
\hline \multicolumn{3}{|c|}{$D_{3}=\left[0,1-\left(1+\alpha_{3}+\alpha_{2} \alpha_{3}\right) \rho_{3}\right) \cap\left(1-\frac{\left(1+\alpha_{1}+\alpha_{1} \alpha_{3}\right) \rho_{3}}{\alpha_{1}}, 1-\frac{\left(1+\alpha_{1}+\alpha_{1} \alpha_{3}\right) \rho_{3}}{\alpha_{1}+\rho_{3}}\right]$} \\
\hline
\end{tabular}

Theorem 7.2 Let $u$ be such that $\alpha_{1} \alpha_{2} \alpha_{3} \leq 1$. Consider a sequence of recognition probability vectors $\left(\rho^{n}\right)_{n \in \mathbb{N}}$ which converges to $e^{i}$, the $i$-th unit vector, for some $i=1,2$, 3. For $n \in \mathbb{N}$, let $\left(p^{n}, a^{n}\right)$ be an SSPE of $\left(u, \rho^{n}\right)$ and denote the corresponding delay probability by $\delta_{n}$. Then $\lim _{n \rightarrow \infty} \delta_{n}=1$.

Theorem 7.2 complements Theorem 7.1 and shows that extreme SSPE delay may occur for certain distributions of bargaining power. To explain the result of Theorem 7.2, it should be recalled that SSPE continuation payoffs are always less than or equal to $u_{m_{i}}^{i}$. Assume player $i$ has $\rho_{i}$ close to one. When he proposes his first-best, it should be turned down with probability close to 1 , to avoid his continuation payoff reaching values above $u_{m_{i}}^{i}$. His continuation payoff will actually be equal to $u_{m_{i}}^{i}$ in an SSPE, implying that player $i$ proposes his best alternative with probability 1 and his middle alternative with probability 0 . On the equilibrium path it therefore holds that player $i$ is recognized as a proposer almost all the time, he proposes his best alternative with probability 1 , which is subsequently turned down with probability close to 1 . The probability of delay is therefore close to 1 in every bargaining round.

We also analyze whether cycles can occur. Cycles should occur according to the Condorcet logic. Other authors like Chwe (1994) have argued using tools from cooperative game theory that cycles should not occur when players are farsighted. We say 
that a particular play of the game has resulted in a cycle if all three alternatives have been proposed and rejected, before some alternative is accepted. An SSPE is said to have a cycle if there is a positive probability that the equilibrium path has resulted in a cycle. If an SSPE has a cycle, then clearly there is also a positive probability on an equilibrium path where consecutively alternatives 1,2,3, and 1 are proposed and rejected.

Theorem 7.3 The set of games $(u, \rho) \in \mathcal{G}$ admitting SSPEs with cycles has Lebesgue measure zero.

Cycles occur with positive probability in the SSPEs of Theorem A.1 and A.4.3. It is easily verified that for Theorem A.1 there is always an SSPE where the equilibrium path results in a cycle with probability arbitrarily close to one. But the conditions of these theorems hold in degenerate cases only.

\section{Comparative statics: recognition probabilities and risk coefficients}

In this section we investigate the comparative statics of the SSPE outcome probability vector $\left(\bar{\pi}_{1}, \bar{\pi}_{2}, \bar{\pi}_{3}\right)$ induced by changes in the recognition probabilities $\rho$ and the risk coefficients $\alpha$. We say that a change in a parameter value weakly improves the bargaining position of a player $i$ if $\bar{\pi}_{b_{i}}^{\prime} \geq \bar{\pi}_{b_{i}}$ and $\bar{\pi}_{w_{i}}^{\prime} \leq \bar{\pi}_{w_{i}}$, where $\left(\bar{\pi}_{1}^{\prime}, \bar{\pi}_{2}^{\prime}, \bar{\pi}_{3}^{\prime}\right)$ is the SSPE outcome probability vector after the change and $\left(\bar{\pi}_{1}, \bar{\pi}_{2}, \bar{\pi}_{3}\right)$ is the SSPE outcome probability vector before the change.

The bargaining position of player $i$ is said to be improved if one of the two inequalities is strict. The induced order on outcome probability distributions is not complete as it remains silent when the probability of obtaining the best outcome and the probability of obtaining the worst outcome move in the same direction.

Table 5 reports the SSPE outcome probability vectors. For Theorem A.4.3, first line, $\lambda \geq 1$ should be chosen to satisfy

$$
\frac{\alpha_{2}}{1+\alpha_{2}+\alpha_{1} \alpha_{2}}<\lambda \rho_{1} \leq \frac{\alpha_{2}+\rho_{1}}{1+\alpha_{2}+\alpha_{1} \alpha_{2}}
$$

and

$$
\lambda \rho_{1}<\frac{1}{1+\alpha_{1}+\alpha_{1} \alpha_{3}} .
$$

For the other two lines corresponding to Theorem A.4.3, $\lambda \geq 1$ should satisfy the appropriate analogues of these inequalities.

We start by evaluating the local effects of a change in a player's recognition probability. We do so under the assumption that an increase in the recognition probability of player $i$ leads to a proportional decrease in the recognition probability of the other two players. We restrict attention to the generic parameter values of Theorems A.3.1, A.3.2, A.4.2, and A.4.4 for which SSPE outcome probability vectors are unique.

It is immediate from Table 5 that a change in recognition probabilities has no effect whatsoever on the outcome probability vector for the case of Theorem A.3.1. The 


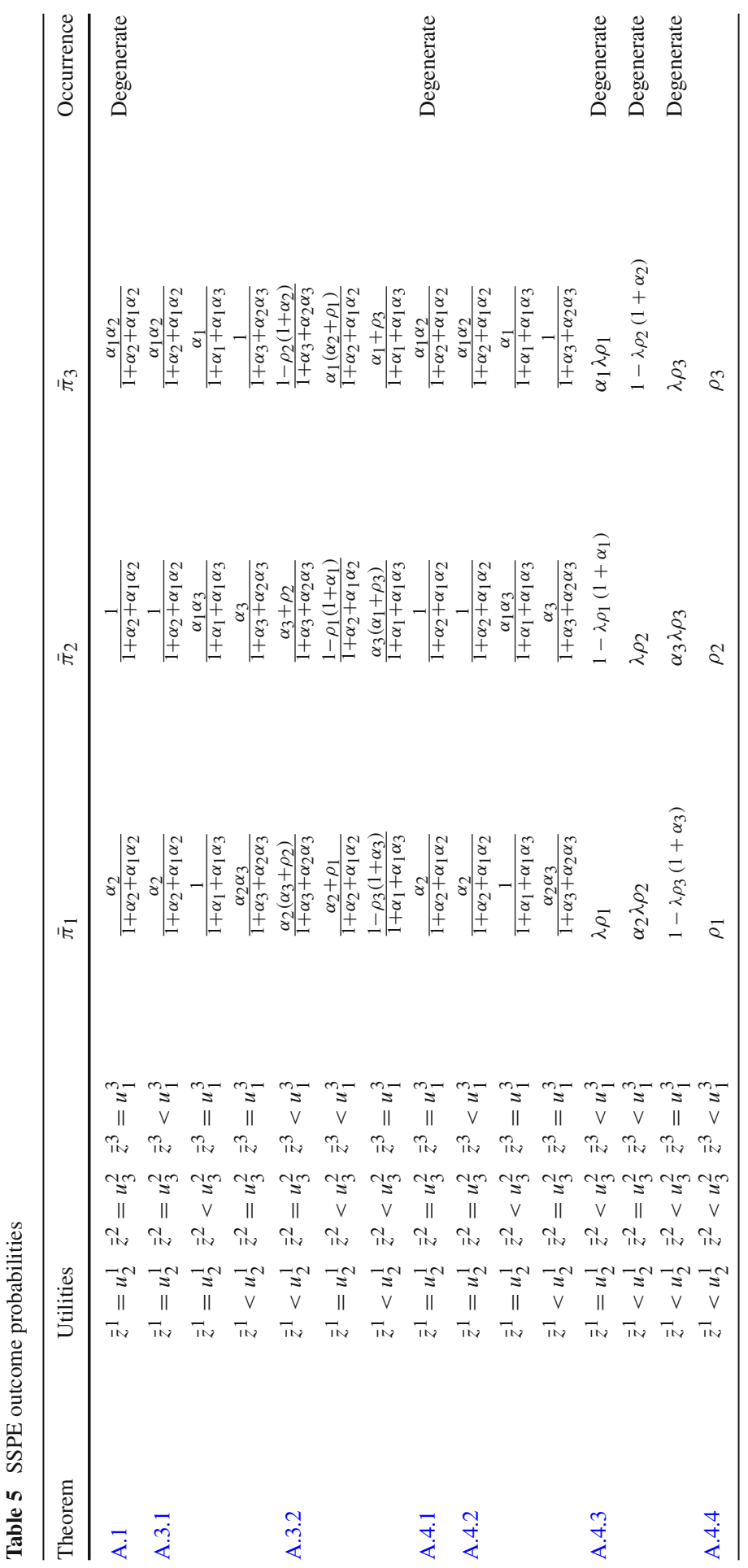


equilibrium type of Theorem A.3.1 is such that two players have a continuation value equal to the utility of their middle alternative. This completely pins down the equilibrium probabilities by which the three outcomes occur, i.e. the outcome probability vector. Exactly the same reasoning applies to the case of Theorem A.4.2.

Next, consider the first line of Theorem A.3.2. The vector of outcome probabilities does not depend on $\rho_{1}$ and $\rho_{3}$, but only on $\rho_{2}$. The reason is that in equilibrium players 1 and 2 propose their best alternative with probability 1 , and player 3 randomizes between proposing $x_{1}$ and $x_{3}$. Moreover, the expected equilibrium utility $\bar{z}^{2}$ of player 2 is equal to $u_{3}^{2}$, the utility of his middle alternative. If $\rho_{2}$ does not change, then $\bar{\pi}_{2}$ does not change, since player 2 is the only player proposing $x_{2}$. To keep the utility of player 2 equal to $u_{3}^{2}$, it follows that $\bar{\pi}_{1}$ should not change, the probability that player 2 gets his worst alternative. Since perpetual delay does not occur, it follows that also $\bar{\pi}_{3}$ does not change. An increase in $\rho_{2}$ leads to a higher probability $\bar{\pi}_{2}$, so to keep the equilibrium utility $\bar{z}^{2}$ of player 2 equal to $u_{3}^{2}, \bar{\pi}_{1}$ has to go up as well. Then $\bar{\pi}_{3}$ has to go down.

If we consider the comparative statics of an increase in the recognition probability $\rho_{1}$ of player 1 accompanied by a proportional decrease in $\rho_{2}$ and $\rho_{3}$, then the decrease in $\rho_{2}$ causes $\bar{\pi}_{1}$ and $\bar{\pi}_{2}$ to go down and $\bar{\pi}_{3}$ to go up. This leads to counterintuitive comparative statics, where a decrease in recognition probability for player 1 improves his bargaining position. An increase in $\rho_{2}$ increases $\bar{\pi}_{1}$ and $\bar{\pi}_{2}$ and decreases $\bar{\pi}_{3}$, so the probability that player 2 receives his best and his worst alternative go both up in such a way that the equilibrium utility of player 2 remains equal to $u_{3}^{2}$. Finally, an increase in $\rho_{3}$ accompanied by a proportional decrease in $\rho_{1}$ and $\rho_{2}$ decreases $\bar{\pi}_{1}$ and $\bar{\pi}_{2}$ and increases $\bar{\pi}_{3}$, so improves the bargaining position of player 3 . The second and third line corresponding to Theorem A.3.2 are obtained by a permutation of the players and lead to analogous results.

The case of Theorem A.4.4 is straightforward. Since every player proposes his best alternative with probability 1 , the outcome probability vector $\bar{\pi}$ is equal to $\rho$. The comparative statics of changes in $\rho$ are completely intuitive.

In summary, we obtain for the cases of Theorems A.3.1 and A.4.2 that changes in the recognition probabilities have no effect on the outcome probability vector. Theorem A.3.2 leads to the counterintuitive result that an increased recognition probability weakens the bargaining position. Finally, Theorem A.4.4 gives the standard result that an increased recognition probability improves the bargaining position.

We continue with evaluating the local effects of a change in a player's risk coefficient. We again restrict attention to the generic parameter values of Theorems A.3.1, A.3.2, A.4.2, and A.4.4 for which SSPE outcome probability vectors are unique. An increase in $\alpha_{i}$ corresponds to an increase in the utility difference of the best and the middle alternative of player $i$ relative to the utility difference between the middle and the worst alternative of player $i$. We find that lower risk coefficients lead to a weakly improved bargaining position.

Consider the first line of Theorems A.3.1 and A.4.2 for which

$$
\left(\bar{\pi}_{1}, \bar{\pi}_{2}, \bar{\pi}_{3}\right)=\left(\frac{\alpha_{2}}{1+\alpha_{2}+\alpha_{1} \alpha_{2}}, \frac{1}{1+\alpha_{2}+\alpha_{1} \alpha_{2}}, \frac{\alpha_{1} \alpha_{2}}{1+\alpha_{2}+\alpha_{1} \alpha_{2}}\right) \text {. }
$$

We have that 


$$
\begin{array}{lll}
\frac{\partial \bar{\pi}_{1}}{\partial \alpha_{1}}<0, & \frac{\partial \bar{\pi}_{2}}{\partial \alpha_{1}}<0, & \frac{\partial \bar{\pi}_{3}}{\partial \alpha_{1}}>0, \\
\frac{\partial \bar{\pi}_{1}}{\partial \alpha_{2}}>0, & \frac{\partial \bar{\pi}_{2}}{\partial \alpha_{2}}<0, & \frac{\partial \bar{\pi}_{3}}{\partial \alpha_{2}}>0, \\
\frac{\partial \bar{\pi}_{1}}{\partial \alpha_{3}}=0, & \frac{\partial \bar{\pi}_{2}}{\partial \alpha_{3}}=0, & \frac{\partial \bar{\pi}_{3}}{\partial \alpha_{3}}=0,
\end{array}
$$

and $\partial \bar{\pi}_{1} / \partial \alpha_{i}+\partial \bar{\pi}_{2} / \partial \alpha_{i}+\partial \bar{\pi}_{3} / \partial \alpha_{i}=0$ for $i=1,2,3$.

An increase in $\alpha_{1}$ yields player 1 lower probabilities for obtaining his best and middle alternatives and a higher probability for obtaining his worst alternative, so weakens his bargaining position. Player 3 faces a higher probability for obtaining his best alternative and lower probabilities for obtaining his middle and worst alternatives if $\alpha_{1}$ increases, so his bargaining position is improved. Player 2 faces lower probabilities for both his best and worst alternatives and a higher probability for his middle alternative when $\alpha_{1}$ increases.

Next, an increase in $\alpha_{2}$ yields player 2 a lower probability for obtaining his best alternative and higher probabilities for attaining his middle and worst alternatives, so weakens his bargaining position. Notice that player 2 is affected in a different manner by a change in $\alpha_{2}$ than player 1 is affected by a change in $\alpha_{1}$. Player 3 faces increased probabilities for obtaining his best and middle alternatives and a lower probability for obtaining his worst alternative if player 2 has a higher risk coefficient, so his bargaining position is improved. Player 1 faces higher probabilities for both his best and worst alternatives and an increased probability for his middle alternative when $\alpha_{2}$ increases.

Finally, changes in player 3's risk coefficient have no effect on the outcome probability vector.

The crucial insight for changes in $\alpha_{1}$ and $\alpha_{2}$ is that when at an SSPE a player's continuation value is equal to the utility of his middle alternative, this property is preserved under small changes of the player's utility function. When the player's risk coefficient increases, the only way to keep his continuation utility equal to the utility of the middle alternative, is to lower the probability by which he obtains his best alternative and increase the probability by which he obtains his worst alternative. The effect of a change in $\alpha_{3}$ is more subtle. Since player 1 and 2 have a continuation value equal to the utility of their middle alternative, the ratio of the probability of receiving the best alternative and the probability of receiving the worst alternative should not be affected by a change in $\alpha_{3}$. Since freezing both ratios means that the outcome probability vector does not change, we find that a change in $\alpha_{3}$ has no effects.

The second and third line corresponding to Theorems A.3.1 and A.4.2 are obtained by a permutation of the players and lead to analogous results.

We verify that also in the case of Theorems A.3.2 and A.4.4 lower risk coefficients improve the bargaining position. Consider the first line corresponding to Theorem A.3.2 for which

$$
\left(\bar{\pi}_{1}, \bar{\pi}_{2}, \bar{\pi}_{3}\right)=\left(\frac{\alpha_{2}\left(\alpha_{3}+\rho_{2}\right)}{1+\alpha_{3}+\alpha_{2} \alpha_{3}}, \frac{\alpha_{3}+\rho_{2}}{1+\alpha_{3}+\alpha_{2} \alpha_{3}}, \frac{1-\rho_{2}\left(1+\alpha_{2}\right)}{1+\alpha_{3}+\alpha_{2} \alpha_{3}}\right) .
$$

We have that

$$
\begin{array}{lll}
\frac{\partial \bar{\pi}_{1}}{\partial \alpha_{1}}=0, & \frac{\partial \bar{\pi}_{2}}{\partial \alpha_{1}}=0, & \frac{\partial \bar{\pi}_{3}}{\partial \alpha_{1}}=0, \\
\frac{\partial \bar{\pi}_{1}}{\partial \alpha_{2}}>0, & \frac{\partial \bar{\pi}_{2}}{\partial \alpha_{2}}<0, & \frac{\partial \bar{\pi}_{3}}{\partial \alpha_{2}}<0, \\
\frac{\partial \bar{\pi}_{1}}{\partial \alpha_{3}}>0, & \frac{\partial \bar{\pi}_{2}}{\partial \alpha_{3}}>0, & \frac{\partial \bar{\pi}_{3}}{\partial \alpha_{3}}<0,
\end{array}
$$


and $\partial \bar{\pi}_{1} / \partial \alpha_{1}+\partial \bar{\pi}_{2} / \partial \alpha_{1}+\partial \bar{\pi}_{3} / \partial \alpha_{1}=0$ for $i=1,2,3$. For this derivation, observe that

$$
\frac{\partial}{\partial \alpha_{3}} \frac{\alpha_{2}\left(\alpha_{3}+\rho_{2}\right)}{1+\alpha_{3}+\alpha_{2} \alpha_{3}}=\frac{\alpha_{2}\left(1-\rho_{2}\left(1+\alpha_{2}\right)\right)}{\left(1+\alpha_{3}+\alpha_{2} \alpha_{3}\right)^{2}}=\frac{\alpha_{2}}{1+\alpha_{3}+\alpha_{2} \alpha_{3}} \bar{\pi}_{3}>0
$$

First, changes in player 1's risk coefficient have no effect. Second, in case player 2 has a higher risk coefficient, then this player has a weaker bargaining position and player 1 improves his bargaining position. Third, in case player 3 gets a higher risk coefficient, then this player weakens his bargaining position and player 1 improves his bargaining position. The second and third line corresponding to Theorem A.3.2 are obtained by a permutation of the players and lead to analogous results.

Since $\bar{z}^{2}=u_{3}^{2}$, an increase in $\alpha_{2}$ is detrimental for player 2 by the same reasoning as before. To explain the effect of an increase in $\alpha_{3}$ requires a new insight. Player 1 and 2 propose their best alternative for sure, followed by acceptance with probability 1 , a feature that is preserved following changes in $\alpha_{3}$. Player 3 randomizes as a proposer between his best and his middle alternative, and his continuation utility conditional on being a proposer is equal to $u_{1}^{3}$, a property that is preserved under small changes in $\alpha_{3}$. An increase in $\alpha_{3}$ therefore results in a worse probability mix on outcomes for player 3 conditional on being the proposer, and given unchanged behavior following the recognition of player 1 and 2 as a proposer, this results in a worse ex ante probability mix on outcomes for player 3. Since player 1 plays a pure strategy, a change in $\alpha_{1}$ has no effect.

Consider Theorem A.4.4 for which $\left(\bar{\pi}_{1}, \bar{\pi}_{2}, \bar{\pi}_{3}\right)=\left(\rho_{1}, \rho_{2}, \rho_{3}\right)$. Then changes in any player's risk coefficient have no effect on the outcome probability vector and for each player the situation before and after such a change is unambiguously equivalent. This result is caused by the fact that all players play a pure strategy.

In summary, we obtain for the three cases of Theorems A.3.1, A.3.2, and A.4.2 that a higher risk coefficient weakens the bargaining position in the sense that the probability of attaining the best alternative decreases while the probability for obtaining the worst alternative increases. In the remaining main case, the one of Theorem A.4.4, changes in risk coefficients have no effect. So, combining these four cases, we find that lower risk coefficients weakly improve the bargaining position.

\section{Concluding remarks}

The message that risk-averse players are willing to accept second-best alternatives, thereby defying the Condorcet logic, is valid well beyond the simple case analyzed in this paper. In a general setting with many players and many alternatives, players may accept unfavorable alternatives, if they face the risk of ending up with an outcome that is even worse. It also holds with great generality that the situation where an agreement is never reached cannot be supported in an SSPE. Indeed, such a situation cannot occur whenever there is an outcome that a majority of players prefers over not reaching an agreement, and at least one such player has a positive recognition probability. 
Many SSPEs feature delay before an agreement is reached. We show that for any specification of the players' risk coefficients, there are recognition probability vectors for which no delay occurs before an agreement is reached, but also that expected delay goes to infinity when in the limit a single player is the only proposer. To what extent such results hold in more general settings is an open issue, but our conjecture is that under quite general circumstances players with high bargaining power will be disciplined by the other players by means of frequent rejections of their proposals.

We have argued that a lower risk coefficient weakly improves the bargaining position of a player. This insight is also valid in more general set-ups. Consider an SSPE where a player randomizes between proposing his first best and his second best alternative, so is indifferent between these two proposals. If the player's utility of the first best alternative goes down, then proposing the second best alternative becomes strictly preferred, so to keep the player indifferent, at the new SSPE the probability of an acceptance of the first best should go up when compared to the probability of an acceptance of the second best.

To derive results on existence and generic uniqueness of SSPE for the case with many alternatives and many players may require using different techniques than in this paper, since it is clear that already the case with three players and three alternatives leads to extensive calculations when characterizing the set of SSPEs. Another possibility would be to restrict attention to pure strategy equilibria or to make some assumptions on the primitives like for instance the symmetry assumptions of Section 3. Apart from generalizations in the direction of more alternatives and more players, it is also interesting to study less restrictive notions of equilibrium or to introduce discounting, in particular to understand the case where the geometric average of the players' risk coefficients is above one.

Open Access This article is distributed under the terms of the Creative Commons Attribution 4.0 International License (http://creativecommons.org/licenses/by/4.0/), which permits unrestricted use, distribution, and reproduction in any medium, provided you give appropriate credit to the original author(s) and the source, provide a link to the Creative Commons license, and indicate if changes were made.

\section{Appendix A}

\section{A.1 Three players with $\bar{p}_{m_{i}}^{i}>0$}

Consider a game $(u, \rho) \in \mathcal{G}$ and let $\left(\left(\bar{p}_{b_{i}}^{i}, \bar{p}_{m_{i}}^{i}, \bar{a}_{m_{i}}^{i}, \bar{z}^{i}\right)_{i=1,2,3}, \bar{\pi}\right)$ be a solution to (4.15)-(4.22) with $\bar{p}_{m_{i}}^{i}>0, i=1,2$, 3. From (4.15), for every $i, u_{m_{i}}^{i} \geq \bar{a}_{m_{i-1}}^{i-1} u_{b_{i}}^{i}+$ $\left(1-\bar{a}_{m_{i-1}}^{i-1}\right) \bar{z}^{i}$, so $\bar{z}^{i} \leq u_{m_{i}}^{i}$. We argue next that for every $i, \bar{z}^{i}=u_{m_{i}}^{i}$. Suppose, for some $i, \bar{z}^{i}<u_{m_{i}}^{i}$. Then $\bar{a}_{m_{i}}^{i}=1$ by (4.17), so $\bar{p}_{m_{i+1}}^{i+1}=0$ by (4.15), a contradiction since we are considering the case $\bar{p}_{m_{i+1}}^{i+1}>0$. It follows that for every $i, \bar{z}^{i}=u_{m_{i}}^{i}$, and by (4.15), $\bar{a}_{m_{i-1}}^{i-1}=0$, so the proposal $x_{m_{i}}$ by a player $i$ is accepted with probability 1 and the proposal $x_{b_{i}}$ by a player $i$ is rejected with probability 1 . Note that since a proposal $x_{b_{i}}$ by player $i$ is rejected for sure, player $i$ is indifferent between making such a 
proposal and giving up the right to propose, i.e. propose $x_{0}$. Equations (4.19)-(4.20) now reduce to the system

$$
\begin{aligned}
\bar{\pi}_{1} u_{1}^{1}+\bar{\pi}_{2} u_{2}^{1}+\bar{\pi}_{3} u_{3}^{1} & =u_{2}^{1}, \\
\bar{\pi}_{1} u_{1}^{2}+\bar{\pi}_{2} u_{2}^{2}+\bar{\pi}_{3} u_{3}^{2} & =u_{3}^{2}, \\
\bar{\pi}_{1} u_{1}^{3}+\bar{\pi}_{2} u_{2}^{3}+\bar{\pi}_{3} u_{3}^{3} & =u_{1}^{3}, \\
\bar{\pi}_{1}+\bar{\pi}_{2}+\bar{\pi}_{3} & =1 .
\end{aligned}
$$

Equations (4.21) and (4.22) can be simplified to

$$
\bar{\pi}_{1}: \bar{\pi}_{2}: \bar{\pi}_{3}=\rho_{3} \bar{p}_{m_{3}}^{3}: \rho_{1} \bar{p}_{m_{1}}^{1}: \rho_{2} \bar{p}_{m_{2}}^{2} .
$$

Whenever utilities are such that (A.1)-(A.4) has a solution $\bar{\pi} \gg 0$, an equilibrium of the type we are looking for in this subsection exists. We derive now under what assumptions on utilities such a solution $\bar{\pi}$ exists. We will show that there is at most one solution, so a solution, if it exists, is unique.

From equalities (A.1) and (A.4), we obtain

$$
\left(1-\bar{\pi}_{2}\right)\left(u_{2}^{1}-u_{3}^{1}\right)=\bar{\pi}_{1}\left(u_{1}^{1}-u_{3}^{1}\right) .
$$

Combining (A.2) and (A.4) leads to

$$
\begin{aligned}
\bar{\pi}_{1}\left(u_{3}^{2}-u_{1}^{2}\right) & =\bar{\pi}_{2}\left(u_{2}^{2}-u_{3}^{2}\right) \\
& =\left(u_{2}^{2}-u_{3}^{2}\right)-\bar{\pi}_{1}\left(u_{2}^{2}-u_{3}^{2}\right) \frac{u_{1}^{1}-u_{3}^{1}}{u_{2}^{1}-u_{3}^{1}},
\end{aligned}
$$

where the second equality follows using (A.6). Rewriting the last equality leads to

$$
\bar{\pi}_{1}=\frac{\alpha_{2}}{1+\alpha_{2}+\alpha_{1} \alpha_{2}} .
$$

It is immediate that $0<\bar{\pi}_{1}<1$.

By (A.7) we have $\bar{\pi}_{2}=\bar{\pi}_{1} / \alpha_{2}$, and we find that

$$
\bar{\pi}_{2}=\frac{1}{1+\alpha_{2}+\alpha_{1} \alpha_{2}} .
$$

Since $\bar{\pi}_{3}=1-\bar{\pi}_{1}-\bar{\pi}_{2}$, we find that

$$
\bar{\pi}_{3}=\frac{\alpha_{1} \alpha_{2}}{1+\alpha_{2}+\alpha_{1} \alpha_{2}} .
$$

Obviously, it holds that $0<\bar{\pi}_{2}<1$ and $0<\bar{\pi}_{3}<1$. At this point we have established that there is at most one solution to (A.1)-(A.4). For there to be some solution, (A.3) should hold. Using the already derived expressions for $\bar{\pi}_{1}, \bar{\pi}_{2}$, and $\bar{\pi}_{3}$, we find that (A.3) holds if and only if $\alpha_{1} \alpha_{2} \alpha_{3}=1$. 
A game $(u, \rho)$ with $\alpha_{1} \alpha_{2} \alpha_{3}=1$ has many equilibria $(\bar{p}, \bar{a})$ of the type described in this subsection. All such equilibria can be constructed as follows. Let $\bar{\pi}$ be the uniquely determined probabilities by which the alternatives are implemented at equilibrium. Let $\lambda>0$ be such that, for $i=1,2,3, \lambda \bar{\pi}_{m_{i}} \leq \rho_{i}$. If player $i$ is selected as proposer, he proposes $x_{m_{i}}$ with probability $\lambda \bar{\pi}_{m_{i}} / \rho_{i}$ and $x_{b_{i}}$ with probability $1-\lambda \bar{\pi}_{m_{i}} / \rho_{i}$. The former proposal is accepted, the latter rejected. This construction ensures that (A.5) holds. The higher $\lambda$, the less delay before an alternative is accepted. The highest possible choice of $\lambda$ occurs when there is at least one player $i$ for which $\bar{p}_{m_{i}}^{i}=1$. In that case, the selection of player $i$ as a proposer leads to a proposal that is accepted for sure.

Summarizing, we have the following. Let utilities be such that $\alpha_{1} \alpha_{2} \alpha_{3}=1$, so there is a unique solution $\bar{\pi} \gg 0$ to (A.1)-(A.4). Then the set of SSPEs with all players making a proposal with probability one is given by

$$
\begin{aligned}
\bar{p} & =\left[\begin{array}{ccc}
1-\bar{p}_{m_{1}}^{1} & 0 & \bar{p}_{m_{3}}^{3} \\
\bar{p}_{m_{1}}^{1} & 1-\bar{p}_{m_{2}}^{2} & 0 \\
0 & \bar{p}_{m_{2}}^{2} & 1-\bar{p}_{m_{3}}^{3}
\end{array}\right] \\
\bar{a}^{1} & =\left[\begin{array}{l}
1 \\
0 \\
0
\end{array}\right], \quad \bar{a}^{2}=\left[\begin{array}{l}
0 \\
1 \\
0
\end{array}\right], \quad \text { and } \bar{a}^{3}=\left[\begin{array}{l}
0 \\
0 \\
1
\end{array}\right],
\end{aligned}
$$

where $\bar{p}$ satisfies (A.5). The other SSPEs are obtained by transferring part or all of the probability $1-\bar{p}_{m_{i}}^{i}$ by which $x_{b_{i}}$ is proposed by player $i$ to the option not to make a proposal, $x_{0}$.

In this SSPE, each player randomizes between his security utility $u_{m_{i}}^{i}$, knowing it will be accepted for sure by player $i+1$, and some gamble among all three alternatives in case he either proposes his best alternative, knowing the latter will be rejected for sure, or does not make a proposal at all. In this gamble, at some future date either player $i-1$ proposes player $i$ 's best alternative and player $i$ accepts, or player $i+1$ may propose player $i$ 's worst alternative but since this is player $i-1$ 's best alternative the latter player accepts, or player $i$ proposes his middle alternative, which is accepted by player $i+1$. Notice that all SSPEs are symmetric whenever the recognition probabilities $\left(\rho_{1}, \rho_{2}, \rho_{3}\right)$ are identical to $\left(\bar{\pi}_{2}, \bar{\pi}_{3}, \bar{\pi}_{1}\right)$, because the latter ensures that the probabilities of proposing the middle alternative are equal. In that case there is one SSPE without delay, i.e. $p_{m_{i}}^{i}=1$ for every player $i$. All other SSPEs involve delay.

All SSPEs $(\bar{p}, \bar{a})$ lead to the same equilibrium payoffs $\bar{z}$ given by $\bar{z}^{i}=u_{m_{i}}^{i}$. We have uniqueness in equilibrium utilities but multiplicity in the supporting equilibrium strategies. Since also $\bar{v}_{i}^{i}=u_{m_{i}}^{i}=\bar{z}^{i}$, there is no advantage in being the proposer. The recognition probabilities $\rho$ do not influence the probability $\bar{\pi}_{j}$ that the bargaining process ends with alternative $x_{j}$. These probabilities depend on the utilities only.

We summarize our findings in the following theorem.

Theorem A.1 There is an $\operatorname{SSPE}(\bar{p}, \bar{a}) \in P \times A$ with $\bar{p}_{m_{i}}^{i}>0, i=1,2,3$, if and only if $\alpha_{1} \alpha_{2} \alpha_{3}=1$. In this case, there is a unique SSPE with minimal expected delay, given by the solution $(\bar{p}, \bar{a})$ to (A.5), (A.8), and (A.9) with $\bar{p}_{m_{i}}^{i}=1$ for at least one player $i$, where $\left(\bar{\pi}_{1}, \bar{\pi}_{2}, \bar{\pi}_{3}\right)$ is the unique solution to (A.1)-(A.4). Other SSPEs 
are obtained by proportionally lowering $\bar{p}_{m_{i}}^{i}$ across players $i$, as well as by shifting probability weight from $\bar{p}_{b_{i}}^{i}$ to $\bar{p}_{0}^{i}$. All SSPEs induce the same equilibrium utilities, given by $\bar{z}^{i}=u_{m_{i}}^{i}, i=1,2,3$.

\section{A.2 Two players with $\bar{p}_{m_{i}}^{i}>0$.}

Next we consider SSPEs where one player, without loss of generality player 1, proposes his best alternative for sure, and the other two players put positive weight on their middle alternative. We argue that no such SSPEs exist.

Consider a game $(u, \rho) \in \mathcal{G}$ and let $\left(\left(\bar{p}_{b_{i}}^{i}, \bar{p}_{m_{i}}^{i}, \bar{a}_{m_{i}}^{i}, \bar{z}^{i}\right)_{i=1,2,3}, \bar{\pi}\right)$ be a solution to (4.15)-(4.22) with $\bar{p}_{1}^{1}=1, \bar{p}_{3}^{2}>0$, and $\bar{p}_{1}^{3}>0$. By (4.15), $\bar{a}_{2}^{1} u_{2}^{2}+\left(1-\bar{a}_{2}^{1}\right) \bar{z}^{2} \leq u_{3}^{2}$, so $\bar{z}^{2} \leq u_{3}^{2}$. Suppose $\bar{z}^{2}<u_{3}^{2}$. Then $\bar{a}_{3}^{2}=1$ by (4.17), so $\bar{p}_{1}^{3}=0$ by (4.15), a contradiction to $\bar{p}_{1}^{3}>0$. It follows that $\bar{z}^{2}=u_{3}^{2}$. Now (4.15) implies $u_{3}^{2} \geq \bar{a}_{2}^{1} u_{2}^{2}+\left(1-\bar{a}_{2}^{1}\right) u_{3}^{2}$, so $\bar{a}_{2}^{1}=0$. It follows that if player 2 proposes his best alternative, it is rejected for sure. No other player ever proposes this alternative. The bargaining process never ends with outcome $x_{2}$, i.e. $\bar{\pi}_{2}=0$. This is a contradiction to $\bar{\pi}_{2}>0$.

Theorem A.2 There is no $\operatorname{SSPE}(\bar{p}, \bar{a}) \in P \times A$ with for some $i=1,2,3, \bar{p}_{m_{i}}^{i}=$ $0, \bar{p}_{m_{i-1}}^{i-1}>0$, and $\bar{p}_{m_{i+1}}^{i+1}>0$.

\section{A.3 One player with $\bar{p}_{m_{i}}^{i}>0$.}

Now we consider SSPEs with two players proposing their best alternative for sure, and where one player, without loss of generality player 3, puts positive weight on his middle alternative.

Consider a game $(u, \rho) \in \mathcal{G}$ and let $\left(\left(\bar{p}_{b_{i}}^{i}, \bar{p}_{m_{i}}^{i}, \bar{a}_{m_{i}}^{i}, \bar{z}^{i}\right)_{i=1,2,3}, \bar{\pi}\right)$ be a solution to (4.15)-(4.22) with $\bar{p}_{1}^{1}=1, \bar{p}_{2}^{2}=1$, and $\bar{p}_{1}^{3}>0$. So, player 1 proposes $x_{1}$, player 2 proposes $x_{2}$, and player 3 mixes over $x_{3}$ and $x_{1}$. To obtain $\bar{\pi}_{j}>0, j=1,2,3$, we must have $\bar{p}_{3}^{3}>0, \bar{a}_{2}^{1}>0$, and $\bar{a}_{3}^{2}>0$. By (4.15) and (4.16) we find

$$
\begin{aligned}
& \bar{a}_{1}^{3} u_{1}^{1}+\left(1-\bar{a}_{1}^{3}\right) \bar{z}^{1} \geq u_{2}^{1}, \\
& \bar{a}_{2}^{1} u_{2}^{2}+\left(1-\bar{a}_{2}^{1}\right) \bar{z}^{2} \geq u_{3}^{2}, \\
& \bar{a}_{3}^{2} u_{3}^{3}+\left(1-\bar{a}_{3}^{2}\right) \bar{z}^{3}=u_{1}^{3} .
\end{aligned}
$$

Since $\bar{a}_{3}^{2}>0$ and $u_{3}^{3}>u_{1}^{3}$, the equality implies $\bar{a}_{3}^{2} \in(0,1)$ and $\bar{z}^{3}<u_{1}^{3}$. It follows by (4.17) and (4.18) that $\bar{z}^{2}=u_{3}^{2}$. Since $\bar{z}^{2}=u_{3}^{2}$, the second inequality above is satisfied. Since $\bar{z}^{3}<u_{1}^{3}$, we have $\bar{a}_{1}^{3}=1$ by (4.17), so the proposal of player 1 is accepted for sure. Also, since $\bar{a}_{1}^{3}=1$, the first inequality above is satisfied. By $\bar{a}_{2}^{1}>0$ and (4.18), we also must have $\bar{z}^{1} \leq u_{2}^{1}$. There are now two possible cases. Case 1 where $\bar{z}^{1}=u_{2}^{1}$ and Case 2 with $\bar{z}^{1}<u_{2}^{1}$. In Case 2 we have $\bar{a}_{2}^{1}=1$ by (4.17). 


\section{A.3.1 Case 1}

It holds that $(\bar{p}, \bar{a}) \in P \times A$ is an SSPE if and only if there is $\bar{\pi}$ such that

$$
\begin{aligned}
& \bar{p}=\left[\begin{array}{ccc}
1 & 0 & 1-\bar{p}_{3}^{3} \\
0 & 1 & 0 \\
0 & 0 & \bar{p}_{3}^{3}
\end{array}\right] \\
& \bar{a}^{1}=\left[\begin{array}{c}
1 \\
\bar{a}_{2}^{1} \\
0
\end{array}\right], \quad \bar{a}^{2}=\left[\begin{array}{c}
0 \\
1 \\
\bar{a}_{3}^{2}
\end{array}\right], \quad \text { and } \bar{a}^{3}=\left[\begin{array}{l}
1 \\
0 \\
1
\end{array}\right], \\
& \bar{a}_{3}^{2} u_{3}^{3}+\left(1-\bar{a}_{3}^{2}\right)\left(\bar{\pi}_{1} u_{1}^{3}+\bar{\pi}_{2} u_{2}^{3}+\bar{\pi}_{3} u_{3}^{3}\right)=u_{1}^{3}, \\
& \bar{\pi}_{1} u_{1}^{1}+\bar{\pi}_{2} u_{2}^{1}+\bar{\pi}_{3} u_{3}^{1}=u_{2}^{1}, \\
& \bar{\pi}_{1} u_{1}^{2}+\bar{\pi}_{2} u_{2}^{2}+\bar{\pi}_{3} u_{3}^{2}=u_{3}^{2}, \\
& \bar{\pi}_{1} u_{1}^{3}+\bar{\pi}_{2} u_{2}^{3}+\bar{\pi}_{3} u_{3}^{3}<u_{1}^{3}, \\
& \bar{\pi}_{1}+\bar{\pi}_{2}+\bar{\pi}_{3}=1, \\
& \bar{\pi}_{1}: \bar{\pi}_{2}: \bar{\pi}_{3}=\rho_{1}+\rho_{3}\left(1-\bar{p}_{3}^{3}\right): \rho_{2} \bar{a}_{2}^{1}: \rho_{3} \bar{p}_{3}^{3} \bar{a}_{3}^{2},
\end{aligned}
$$

where $0<\bar{p}_{3}^{3}<1,0<\bar{a}_{2}^{1} \leq 1$, and $0<\bar{a}_{3}^{2}<1$.

Using the same derivation as in Appendix A.1, it can be shown that there is a solution $\bar{\pi} \gg 0$ to the system (A.13)-(A.16) if and only if

$$
\alpha_{1} \alpha_{2} \alpha_{3}<1
$$

Moreover, each specification of utilities satisfying (A.18) leads to a unique solution $\bar{\pi} \gg 0$ to (A.13)-(A.16). Indeed, as before it holds that

$$
\begin{aligned}
& \bar{\pi}_{1}=\frac{\alpha_{2}}{1+\alpha_{2}+\alpha_{1} \alpha_{2}}, \\
& \bar{\pi}_{2}=\frac{1}{1+\alpha_{2}+\alpha_{1} \alpha_{2}}, \\
& \bar{\pi}_{3}=\frac{\alpha_{1} \alpha_{2}}{1+\alpha_{2}+\alpha_{1} \alpha_{2}} .
\end{aligned}
$$

Rewriting (A.12), we obtain

$$
\bar{a}_{3}^{2}=\frac{\bar{\pi}_{2}-\alpha_{3} \bar{\pi}_{3}}{\alpha_{3}+\bar{\pi}_{2}-\alpha_{3} \bar{\pi}_{3}},
$$

and substitution of the expressions for $\bar{\pi}_{2}$ and $\bar{\pi}_{3}$ results in

$$
\bar{a}_{3}^{2}=\frac{1-\alpha_{1} \alpha_{2} \alpha_{3}}{1+\alpha_{3}+\alpha_{2} \alpha_{3}} .
$$


Notice that $0<\bar{a}_{3}^{2}<1$. By (A.17) we have

$$
\alpha_{1}=\frac{\rho_{3} \bar{p}_{3}^{3} \bar{a}_{3}^{2}}{\rho_{1}+\rho_{3}\left(1-\bar{p}_{3}^{3}\right)} .
$$

Substitution of the expression for $\bar{a}_{3}^{2}$ in the latter equation, and then solving for $\bar{p}_{3}^{3}$ results in

$$
\bar{p}_{3}^{3}=\frac{\rho_{1}+\rho_{3}}{\rho_{3}} \frac{\alpha_{1}+\alpha_{1} \alpha_{3}+\alpha_{1} \alpha_{2} \alpha_{3}}{1+\alpha_{1}+\alpha_{1} \alpha_{3}} .
$$

Obviously, it holds that $\bar{p}_{3}^{3}>0$. Moreover, we have that $\bar{p}_{3}^{3}<1$ if and only if

$$
\frac{\rho_{1}}{\rho_{3}}<\frac{1-\alpha_{1} \alpha_{2} \alpha_{3}}{\alpha_{1}+\alpha_{1} \alpha_{3}+\alpha_{1} \alpha_{2} \alpha_{3}} .
$$

By (A.17) we have

$$
\alpha_{2}=\frac{\rho_{1}+\rho_{3}\left(1-\bar{p}_{3}^{3}\right)}{\rho_{2} \bar{a}_{2}^{1}} .
$$

We substitute the expression found for $\bar{p}_{3}^{3}$ and solve the resulting equation for $\bar{a}_{2}^{1}$, and obtain that

$$
\bar{a}_{2}^{1}=\frac{\rho_{1}+\rho_{3}}{\rho_{2}} \frac{1-\alpha_{1} \alpha_{2} \alpha_{3}}{\alpha_{2}+\alpha_{1} \alpha_{2}+\alpha_{1} \alpha_{2} \alpha_{3}} .
$$

This expression is clearly positive. It is less than or equal to one if and only if

$$
\rho_{2} \geq \frac{1-\alpha_{1} \alpha_{2} \alpha_{3}}{1+\alpha_{2}+\alpha_{1} \alpha_{2}}
$$

Since every player $i$ proposes his best alternative $x_{b_{i}}$ with positive probability, and since $\bar{a}_{1}^{3}=1, \bar{a}_{2}^{1}>0$, and $\bar{a}_{3}^{2}>0$ implies that such a proposal is accepted with positive probability, no player wants to use the option not to make a proposal. Finally, the SSPE utilities satisfy $\bar{z}^{1}=u_{2}^{1}, \bar{z}^{2}=u_{3}^{2}$, and $\bar{z}^{3}<u_{1}^{3}$.

We summarize our findings in the following theorem.

Theorem A.3.1 There is an $\operatorname{SSPE}(\bar{p}, \bar{a}) \in P \times A$ with $\bar{p}_{2}^{1}=0, \bar{p}_{3}^{2}=0, \bar{p}_{1}^{3}>0$, and $\bar{z}^{1} \geq u_{2}^{1}$ if and only if $\alpha_{1} \alpha_{2} \alpha_{3}<1$ and $\rho$ is such that (A.21) and (A.23) are satisfied. In this case, such SSPE is unique. It is given by (A.10), (A.11), (A.19), (A.20), and (A.22). The equilibrium utilities satisfy $\bar{z}^{1}=u_{2}^{1}, \bar{z}^{2}=u_{3}^{2}$, and $\bar{z}^{3}<u_{1}^{3}$.

Notice that, unlike the SSPEs of Theorem A.1, players never use the option to refrain from making a proposal, i.e., $p_{0}^{i}=0, i=1,2$, 3. Since conditional equilibrium utilities satisfy $\bar{v}_{i}^{i}>\bar{z}^{i}, i=1,2,3$, each player enjoys an advantage whenever he is the recognized player. Moreover, conditional on being the recognized player, player 1 achieves his best alternative, player 2 is strictly better off compared to his security level, and player 3 is kept at his security level. In many bargaining models, the advantage to propose vanishes in taking the limit to the no discounting case. Here the advantage is present under no discounting. 
The SSPE leads to a positive expected delay. The reason is that player 3 is recognized with positive probability and proposes $x_{3}$ with positive probability. This proposal is rejected by both players with positive probability. Player 1 always proposes $x_{1}$, which is accepted by player 3 . Player 2 always proposes $x_{2}$, which is accepted by player 1 with positive probability $\bar{a}_{2}^{1}$ and is rejected by both players otherwise.

Using a straightforward relabeling of the players, we find fully symmetric results for SSPEs with $\bar{p}_{2}^{2}=\bar{p}_{3}^{3}=1$ and player 1 mixing between $x_{1}$ and $x_{2}$, and SSPEs with $\bar{p}_{1}^{1}=\bar{p}_{3}^{3}=1$ and player 2 mixing between $x_{2}$ and $x_{3}$.

\section{A.3.2 Case 2}

It holds that $(\bar{p}, \bar{a}) \in P \times A$ is an SSPE if and only if there is $\bar{\pi}$ such that

$$
\begin{aligned}
& \bar{p}=\left[\begin{array}{ccc}
1 & 0 & 1-\bar{p}_{3}^{3} \\
0 & 1 & 0 \\
0 & 0 & \bar{p}_{3}^{3}
\end{array}\right] \\
& \bar{a}^{1}=\left[\begin{array}{l}
1 \\
1 \\
0
\end{array}\right], \quad \bar{a}^{2}=\left[\begin{array}{c}
0 \\
1 \\
\bar{a}_{3}^{2}
\end{array}\right], \quad \text { and } \bar{a}^{3}=\left[\begin{array}{l}
1 \\
0 \\
1
\end{array}\right], \\
& \bar{a}_{3}^{2} u_{3}^{3}+\left(1-\bar{a}_{3}^{2}\right)\left(\bar{\pi}_{1} u_{1}^{3}+\bar{\pi}_{2} u_{2}^{3}+\bar{\pi}_{3} u_{3}^{3}\right)=u_{1}^{3}, \\
& \bar{\pi}_{1} u_{1}^{1}+\bar{\pi}_{2} u_{2}^{1}+\bar{\pi}_{3} u_{3}^{1}<u_{2}^{1}, \\
& \bar{\pi}_{1} u_{1}^{2}+\bar{\pi}_{2} u_{2}^{2}+\bar{\pi}_{3} u_{3}^{2}=u_{3}^{2}, \\
& \bar{\pi}_{1} u_{1}^{3}+\bar{\pi}_{2} u_{2}^{3}+\bar{\pi}_{3} u_{3}^{3}<u_{1}^{3}, \\
& \bar{\pi}_{1}+\bar{\pi}_{2}+\bar{\pi}_{3}=1, \\
& \bar{\pi}_{1}: \bar{\pi}_{2}: \bar{\pi}_{3}=\rho_{1}+\rho_{3}\left(1-\bar{p}_{3}^{3}\right): \rho_{2}: \rho_{3} \bar{p}_{3}^{3} \bar{a}_{3}^{2},
\end{aligned}
$$

where $0<\bar{p}_{3}^{3}<1$ and $0<\bar{a}_{3}^{2}<1$.

We can rewrite (A.26)-(A.29) as

$$
\begin{aligned}
\alpha_{3}-\left(1-\bar{a}_{3}^{2}\right)\left(\bar{\pi}_{1} \alpha_{3}+\bar{\pi}_{2}\left(1+\alpha_{3}\right)\right) & =0, \\
\bar{\pi}_{1} \alpha_{1}-\bar{\pi}_{3} & <0, \\
-\bar{\pi}_{1}+\bar{\pi}_{2} \alpha_{2} & =0, \\
-\bar{\pi}_{2}+\bar{\pi}_{3} \alpha_{3} & <0 .
\end{aligned}
$$

We have a system (A.30), (A.31), (A.32), (A.34) with five equations in the five unknowns $\bar{\pi}_{1}, \bar{\pi}_{2}, \bar{\pi}_{3}, \bar{p}_{3}^{3}$, and $\bar{a}_{3}^{2}$. Solving this system results in outcome probabilities

$$
\bar{\pi}_{1}=\frac{\alpha_{2}\left(\alpha_{3}+\rho_{2}\right)}{1+\alpha_{3}+\alpha_{2} \alpha_{3}}, \quad \bar{\pi}_{2}=\frac{\alpha_{3}+\rho_{2}}{1+\alpha_{3}+\alpha_{2} \alpha_{3}}, \quad \text { and } \quad \bar{\pi}_{3}=\frac{1-\rho_{2}\left(1+\alpha_{2}\right)}{1+\alpha_{3}+\alpha_{2} \alpha_{3}} \text {, }
$$


and SSPE action probabilities

$$
\begin{aligned}
\bar{p}_{3}^{3} & =\frac{\rho_{1}-\alpha_{2} \rho_{2}+\rho_{3}}{\rho_{3}}, \\
\bar{a}_{3}^{2} & =\frac{\rho_{2}}{\alpha_{3}+\rho_{2}} .
\end{aligned}
$$

It is immediate that the solution satisfies $0<\bar{a}_{3}^{2}<1$.

The inequality (A.33) is equivalent to

$$
\rho_{2}<\frac{1-\alpha_{1} \alpha_{2} \alpha_{3}}{1+\alpha_{2}+\alpha_{1} \alpha_{2}}\left(<\frac{1}{1+\alpha_{2}}\right) .
$$

The inequality (A.35) is always satisfied.

The requirement $\bar{p}_{3}^{3}>0$ is equivalent to $\rho_{2}<1 /\left(1+\alpha_{2}\right)$, which implies $\bar{\pi}_{3}>0$. This requirement follows from (A.38). The requirement $\bar{p}_{3}^{3}<1$ is equivalent to

$$
\frac{\rho_{1}}{\rho_{2}}<\alpha_{2}
$$

Since every player $i$ proposes his best alternative $x_{b_{i}}$ with positive probability, and since $\bar{a}_{1}^{3}=1, \bar{a}_{2}^{1}=1$, and $\bar{a}_{3}^{2}>0$ implies that such a proposal is accepted with positive probability, no player wants to use the option not to make a proposal.

We summarize our findings in the following theorem.

Theorem A.3.2 There is an $\operatorname{SSPE}(\bar{p}, \bar{a}) \in P \times A$ with $\bar{p}_{2}^{1}=0, \bar{p}_{3}^{2}=0, \bar{p}_{1}^{3}>0$, and $\bar{z}^{1}<u_{2}^{1}$ if and only if $\alpha_{1} \alpha_{2} \alpha_{3}<1$ and $\rho$ is such that (A.38) and (A.39) are satisfied. In this case, an SSPE is unique. It is given by (A.24), (A.25), (A.36), and (A.37). Equilibrium utilities satisfy $\bar{z}^{1}<u_{2}^{1}, \bar{z}^{2}=u_{3}^{2}$, and $\bar{z}^{3}<u_{1}^{3}$.

For given utilities satisfying (A.18), (A.38) requires $\rho_{2}$ to be sufficiently low. It complements (A.23) which implies that SSPEs as in Case 1 cannot coexist with those as in Case 2. Inequality (A.39) requires $\rho_{1}$ to be sufficiently low compared to $\rho_{2}$. Notice that, like the SSPE of Theorem A.3.1, the option not to make a proposal cannot be chosen with any positive probability.

The SSPE leads to a positive expected delay. The reason is that player 3 is recognized with positive probability, proposes $x_{3}$ with positive probability, which is rejected by both players with positive probability. The proposals of players 1 and 2 are accepted for sure. Similar to the previous case, all three players have an advantage to propose. Conditional on being the recognized player, player 3 cannot do better than getting the utility of his middle alternative. Conditional on being the proposer, both player 1 and player 2 achieve the utility of the best alternative.

By a relabeling of the players, we find fully symmetric results for SSPEs with $\bar{p}_{2}^{2}=\bar{p}_{3}^{3}=1$ and player 1 mixing between $x_{1}$ and $x_{2}$, and SSPEs with $\bar{p}_{1}^{1}=\bar{p}_{3}^{3}=1$ and player 2 mixing between $x_{2}$ and $x_{3}$. 


\section{A.4 No player with $\bar{p}_{m_{i}}^{i}>0$}

We finally consider SSPEs where all players propose their best alternative for sure. Let $\left(\bar{p}_{b_{i}}^{i}, \bar{p}_{m_{i}}^{i}, \bar{a}_{m_{i}}^{i}, \bar{z}^{i}\right)$ be a solution to (4.15)-(4.22) with $\bar{p}_{1}^{1}=\bar{p}_{2}^{2}=\bar{p}_{3}^{3}=1$. To obtain $\bar{\pi}_{j}>0, j=1,2,3$, we must have $\bar{a}_{1}^{3}>0, \bar{a}_{2}^{1}>0$, and $\bar{a}_{3}^{2}>0$. If follows from (4.18) that $\bar{z}^{1} \leq u_{2}^{1}, \bar{z}^{2} \leq u_{3}^{2}$, and $\bar{z}^{3} \leq u_{1}^{3}$. Since all players propose their best alternative with positive probability, and since such a proposal is accepted with positive probability, no player wants to use the option not to make a proposal. We distinguish four possible cases of interest. In Case 1, there are three players with $\bar{z}^{i}=u_{m_{i}}^{i}$, in Case 2 there are two such players, without loss of generality, players 1 and 2, in Case 3 there is one such player, without loss of generality player 1, and in Case 4 every player $i$ has $\bar{z}^{i}<u_{m_{i}}^{i}$.

\section{A.4.1 Case 1}

It holds that $(\bar{p}, \bar{a}) \in P \times A$ is an SSPE if and only if there is $\bar{\pi}$ such that

$$
\begin{aligned}
& \bar{p}=\left[\begin{array}{lll}
1 & 0 & 0 \\
0 & 1 & 0 \\
0 & 0 & 1
\end{array}\right] \\
& \bar{a}^{1}=\left[\begin{array}{c}
1 \\
\bar{a}_{2}^{1} \\
0
\end{array}\right], \quad \bar{a}^{2}=\left[\begin{array}{c}
0 \\
1 \\
\bar{a}_{3}^{2}
\end{array}\right], \quad \text { and } \bar{a}^{3}=\left[\begin{array}{c}
\bar{a}_{1}^{3} \\
0 \\
1
\end{array}\right], \\
& \bar{\pi}_{1} u_{1}^{1}+\bar{\pi}_{2} u_{2}^{1}+\bar{\pi}_{3} u_{3}^{1}=u_{2}^{1}, \\
& \bar{\pi}_{1} u_{1}^{2}+\bar{\pi}_{2} u_{2}^{2}+\bar{\pi}_{3} u_{3}^{2}=u_{3}^{2}, \\
& \bar{\pi}_{1} u_{1}^{3}+\bar{\pi}_{2} u_{2}^{3}+\bar{\pi}_{3} u_{3}^{3}=u_{1}^{3}, \\
& \bar{\pi}_{1}+\bar{\pi}_{2}+\bar{\pi}_{3}=1, \\
& \bar{\pi}_{1}: \bar{\pi}_{2}: \bar{\pi}_{3}=\rho_{1} \bar{a}_{1}^{3}: \rho_{2} \bar{a}_{2}^{1}: \rho_{3} \bar{a}_{3}^{2} .
\end{aligned}
$$

As in Appendix A.1 we obtain that

$$
\begin{aligned}
\alpha_{1} \alpha_{2} \alpha_{3} & =1, \\
\bar{\pi}_{1} & =\frac{\alpha_{2}}{1+\alpha_{2}+\alpha_{1} \alpha_{2}}, \\
\bar{\pi}_{2} & =\frac{1}{1+\alpha_{2}+\alpha_{1} \alpha_{2}}, \\
\bar{\pi}_{3} & =\frac{\alpha_{1} \alpha_{2}}{1+\alpha_{2}+\alpha_{1} \alpha_{2}} .
\end{aligned}
$$

The SSPE is not unique. Let $\lambda>0$ be such that, for $i=1,2,3, \lambda \bar{\pi}_{m_{i}} \leq \rho_{m_{i}}$. If player $i$ has to respond to the proposal $x_{m_{i}}$, he accepts with probability $\bar{a}_{m_{i}}^{i}=\lambda \bar{\pi}_{m_{i}} / \rho_{m_{i}}>0$ and rejects with probability $1-\lambda \bar{\pi}_{m_{i}} / \rho_{m_{i}}<1$. This construction ensures that (A.42) holds. The higher $\lambda$, the less delay before an outcome is accepted. The highest possible choice of $\lambda$ occurs when there is at least one player $i$ for which $\bar{a}_{m_{i}}^{i}=1$. In that case, 
selection of player $i+1$ as proposer leads to a proposal that is accepted for sure. Note that $\lambda=0$ would violate $\bar{\pi}_{m_{i}}>0$. The set of SSPEs is not closed. The no discounting case differs in this respect from the discounting case where the set of SSPEs is compact.

By definition of this case, the equilibrium utilities satisfy $\bar{z}^{i}=u_{m_{i}}^{i}, i=1,2,3$. Since also $\bar{a}_{m_{i}}^{i}>0$ and $\bar{z}^{i}=u_{m_{i}}^{i}$, the conditional equilibrium utilities satisfy $\bar{v}_{i}^{i} \in\left(u_{m_{i}}^{i}, u_{b_{i}}^{i}\right), i=1,2,3$. We conclude that there is an advantage in becoming the recognized player and that a recognized player does strictly better than his security level $u_{m_{i}}^{i}$.

Theorem A.4.1 There is an SSPE $(\bar{p}, \bar{a}) \in P \times A$ with $\bar{p}_{m_{i}}^{i}=0, i=1,2,3$, and $\bar{z}^{i} \geq u_{m_{i}}^{i}, i=1,2,3$, if and only if $\alpha_{1} \alpha_{2} \alpha_{3}=1$. In this case, there is a unique SSPE with minimal delay. It is given by the solution $(\bar{p}, \bar{a})$ to (A.40), (A.41), and (A.42) with $\bar{a}_{m_{i}}^{i}=1$ for at least one player $i$, where $\left(\bar{\pi}_{1}, \bar{\pi}_{2}, \bar{\pi}_{3}\right)$ is defined in (A.44)-( A.46). Other SSPEs are obtained by proportionally lowering $\bar{a}_{m_{i}}^{i}$ across players $i$. All SSPEs induce the same equilibrium utilities, given by $\bar{z}^{i}=u_{m_{i}}^{i}, i=1,2,3$.

\section{A.4.2 Case 2}

It holds that $(\bar{p}, \bar{a}) \in P \times A$ is an SSPE if and only if there is $\bar{\pi}$ such that

$$
\begin{aligned}
& \bar{p}=\left[\begin{array}{lll}
1 & 0 & 0 \\
0 & 1 & 0 \\
0 & 0 & 1
\end{array}\right] \\
& \bar{a}^{1}=\left[\begin{array}{c}
1 \\
\bar{a}_{2}^{1} \\
0
\end{array}\right], \quad \bar{a}^{2}=\left[\begin{array}{c}
0 \\
1 \\
\bar{a}_{3}^{2}
\end{array}\right], \quad \text { and } \bar{a}^{3}=\left[\begin{array}{l}
1 \\
0 \\
1
\end{array}\right], \\
& \bar{a}_{3}^{2} u_{3}^{3}+\left(1-\bar{a}_{3}^{2}\right)\left(\bar{\pi}_{1} u_{1}^{3}+\bar{\pi}_{2} u_{2}^{3}+\bar{\pi}_{3} u_{3}^{3}\right) \geq u_{1}^{3}, \\
& \bar{\pi}_{1} u_{1}^{1}+\bar{\pi}_{2} u_{2}^{1}+\bar{\pi}_{3} u_{3}^{1}=u_{2}^{1}, \\
& \bar{\pi}_{1} u_{1}^{2}+\bar{\pi}_{2} u_{2}^{2}+\bar{\pi}_{3} u_{3}^{2}=u_{3}^{2}, \\
& \bar{\pi}_{1} u_{1}^{3}+\bar{\pi}_{2} u_{2}^{3}+\bar{\pi}_{3} u_{3}^{3}<u_{1}^{3}, \\
& \bar{\pi}_{1}+\bar{\pi}_{2}+\bar{\pi}_{3}=1, \\
& \bar{\pi}_{1}: \bar{\pi}_{2}: \bar{\pi}_{3}=\rho_{1}: \rho_{2} \bar{a}_{2}^{1}: \rho_{3} \bar{a}_{3}^{2} .
\end{aligned}
$$

As in Case 1 in Subsects. A.3.1 we obtain that

$$
\begin{aligned}
\alpha_{1} \alpha_{2} \alpha_{3} & <1, \\
\bar{\pi}_{1} & =\frac{\alpha_{2}}{1+\alpha_{2}+\alpha_{1} \alpha_{2}}, \quad \bar{\pi}_{2}=\frac{1}{1+\alpha_{2}+\alpha_{1} \alpha_{2}}, \quad \text { and } \quad \bar{\pi}_{3}=\frac{\alpha_{1} \alpha_{2}}{1+\alpha_{2}+\alpha_{1} \alpha_{2}} .
\end{aligned}
$$


From (A.50) it then follows that

$$
\begin{aligned}
& \bar{a}_{2}^{1}=\frac{\rho_{1}}{\alpha_{2} \rho_{2}}, \\
& \bar{a}_{3}^{2}=\frac{\alpha_{1} \rho_{1}}{\rho_{3}} .
\end{aligned}
$$

To satisfy (A.49) we need that

$$
\frac{\rho_{1}}{\rho_{3}} \geq \frac{1-\alpha_{1} \alpha_{2} \alpha_{3}}{\alpha_{1}+\alpha_{1} \alpha_{3}+\alpha_{1} \alpha_{2} \alpha_{3}}\left(<\frac{1}{\alpha_{1}}\right) .
$$

The requirements $\bar{a}_{2}^{1} \leq 1$ and $\bar{a}_{3}^{2} \leq 1$ lead to

$$
\begin{aligned}
& \frac{\rho_{1}}{\rho_{2}} \leq \alpha_{2}, \\
& \frac{\rho_{3}}{\rho_{1}} \geq \alpha_{1} .
\end{aligned}
$$

By definition of the case, the equilibrium utilities satisfy $\bar{z}^{1}=u_{m_{1}}^{1}, \bar{z}^{2}=u_{m_{2}}^{2}$, and $\bar{z}^{3}<u_{m_{3}}^{3}$. For $i=1,2, \bar{a}_{b_{i}}^{i-1}>0$ and $\bar{z}^{i}=u_{m_{i}}^{i}$ imply that the conditional equilibrium utilities satisfy $\bar{v}_{i}^{i}>u_{m_{i}}^{i}$. Since $\bar{z}^{3}<u_{m_{3}}^{3}$, it follows that player 3 has an advantage to propose.

We summarize our findings in the following theorem.

Theorem A.4.2 There is an $\operatorname{SSPE}(\bar{p}, \bar{a}) \in P \times A$ with $\bar{p}_{m_{i}}^{i}=0, i=1,2,3, \bar{z}^{1} \geq u_{2}^{1}$, $\bar{z}^{2} \geq u_{3}^{2}$, and $\bar{z}^{3}<u_{1}^{3}$ if and only if $\alpha_{1} \alpha_{2} \alpha_{3}<1$ and $\rho$ is such that (A.54), (A.55), and (A.56) are satisfied. In this case, there is a unique SSPE. It is given by (A.47), (A.48), (A.52), and (A.53). The equilibrium utilities satisfy $\bar{z}^{1}=u_{2}^{1}, \bar{z}^{2}=u_{3}^{2}$, and $\bar{z}^{3}<u_{1}^{3}$.

By (A.50), the SSPE does not involve delay if and only if $\rho_{i}$ is equal to $\bar{\pi}_{i}$ for all $i=1,2,3$.

By a relabeling of the players, we obtain fully symmetric results for SSPEs with $\bar{p}_{m_{i}}^{i}=0, i=1,2,3, \bar{z}^{1} \geq u_{2}^{1}, \bar{z}^{2}<u_{3}^{2}$, and $\bar{z}^{3} \geq u_{1}^{3}$, and for SSPEs with $\bar{p}_{m_{i}}^{i}=0$, $i=1,2,3, \bar{z}^{1}<u_{2}^{1}, \bar{z}^{2} \geq u_{3}^{2}$, and $\bar{z}^{3} \geq u_{1}^{3}$.

\section{A.4.3 Case 3}

It holds that $(\bar{p}, \bar{a}) \in P \times A$ is an SSPE if and only if there is $\bar{\pi}$ such that

$$
\begin{aligned}
& \bar{p}=\left[\begin{array}{lll}
1 & 0 & 0 \\
0 & 1 & 0 \\
0 & 0 & 1
\end{array}\right] \\
& \bar{a}^{1}=\left[\begin{array}{c}
1 \\
\bar{a}_{2}^{1} \\
0
\end{array}\right], \quad \bar{a}^{2}=\left[\begin{array}{l}
0 \\
1 \\
1
\end{array}\right], \quad \text { and } \bar{a}^{3}=\left[\begin{array}{l}
1 \\
0 \\
1
\end{array}\right],
\end{aligned}
$$




$$
\begin{aligned}
& \bar{a}_{2}^{1} u_{2}^{2}+\left(1-\bar{a}_{2}^{1}\right)\left(\bar{\pi}_{1} u_{1}^{2}+\bar{\pi}_{2} u_{2}^{2}+\bar{\pi}_{3} u_{3}^{2}\right) \geq u_{3}^{2}, \\
& \bar{\pi}_{1} u_{1}^{1}+\bar{\pi}_{2} u_{2}^{1}+\bar{\pi}_{3} u_{3}^{1}=u_{2}^{1}, \\
& \bar{\pi}_{1} u_{1}^{2}+\bar{\pi}_{2} u_{2}^{2}+\bar{\pi}_{3} u_{3}^{2}<u_{3}^{2}, \\
& \bar{\pi}_{1} u_{1}^{3}+\bar{\pi}_{2} u_{2}^{3}+\bar{\pi}_{3} u_{3}^{3}<u_{1}^{3}, \\
& \bar{\pi}_{1}+\bar{\pi}_{2}+\bar{\pi}_{3}=1, \\
& \bar{\pi}_{1}: \bar{\pi}_{2}: \bar{\pi}_{3}=\rho_{1}: \rho_{2} \bar{a}_{2}^{1}: \rho_{3} .
\end{aligned}
$$

Notice that the analogue of (A.59) for player 3 is automatically satisfied since $\bar{a}_{3}^{2}=1$. Rewriting (A.60) and using (A.63), we find that

$$
\rho_{3}=\alpha_{1} \rho_{1}
$$

It follows that Case 3 admits SSPEs in degenerate cases only, more precisely, when (A.64) holds. In these degenerate cases, there is a continuum of SSPEs, inducing a continuum of SSPE utilities for players 2 and 3. We parametrize the SSPEs by means of the positive real number $\lambda$ and using (A.63) we write

$$
\bar{\pi}_{1}=\lambda \rho_{1} \text { and } \bar{\pi}_{3}=\alpha_{1} \lambda \rho_{1} \text {. }
$$

Suppose by means of contradiction that $\lambda<1$. Using (A.63), we find that

$$
\bar{\pi}_{1}=\lambda \rho_{1}<\rho_{1}, \bar{\pi}_{2}=\frac{\bar{\pi}_{1} \rho_{2} \bar{a}_{2}^{1}}{\rho_{1}}=\lambda \rho_{2} \bar{a}_{2}^{1}<\rho_{2}, \quad \text { and } \quad \bar{\pi}_{3}=\frac{\bar{\pi}_{1} \rho_{3}}{\rho_{1}}=\lambda \rho_{3}<\rho_{3} .
$$

We obtain the contradiction $1=\bar{\pi}_{1}+\bar{\pi}_{2}+\bar{\pi}_{3}<\rho_{1}+\rho_{2}+\rho_{3}=1$. Consequently, we have shown that $\lambda \geq 1$.

Since $\rho_{1}+\rho_{2}+\rho_{3}=1$ and $\bar{\pi}_{1}+\bar{\pi}_{2}+\bar{\pi}_{3}=1$, we have

$$
\rho_{2}=1-\left(1+\alpha_{1}\right) \rho_{1}, \text { and } \bar{\pi}_{2}=1-\left(1+\alpha_{1}\right) \lambda \rho_{1} \text {. }
$$

Using (A.63), we have

$$
\bar{a}_{2}^{1}=\frac{1-\left(1+\alpha_{1}\right) \lambda \rho_{1}}{\lambda-\left(1+\alpha_{1}\right) \lambda \rho_{1}} .
$$

The denominator of (A.65) is positive if and only if $\rho_{1}<1 /\left(1+\alpha_{1}\right)$. The inequalities in (A.61) and (A.59) are satisfied if and only if

$$
\frac{\alpha_{2}}{1+\alpha_{2}+\alpha_{1} \alpha_{2}}<\lambda \rho_{1} \leq \frac{\alpha_{2}+\rho_{1}}{1+\alpha_{2}+\alpha_{1} \alpha_{2}}\left(<\frac{1}{1+\alpha_{1}}\right)
$$

The inequality in (A.66) in parentheses implies that $\bar{a}_{2}^{1}$ and $\bar{\pi}_{2}$ are positive.

The inequality (A.62) is satisfied if and only if

$$
\lambda \rho_{1}<\frac{1}{1+\alpha_{1}+\alpha_{1} \alpha_{3}} .
$$


The first inequality of (A.66) together with (A.67) imply that $\alpha_{1} \alpha_{2} \alpha_{3}<1$. There is some $\lambda \geq 1$ such that (A.66) and (A.67) are satisfied if and only if $\alpha_{1} \alpha_{2} \alpha_{3}<1$ and

$$
\rho_{1}<\frac{1}{1+\alpha_{1}+\alpha_{1} \alpha_{3}}
$$

We summarize our findings in the following theorem.

Theorem A.4.3 There is an SSPE $(\bar{p}, \bar{a}) \in P \times A$ with $\bar{p}_{m_{i}}^{i}=0, i=1,2,3, \bar{z}^{1} \geq u_{2}^{1}$, $\bar{z}^{2}<u_{3}^{2}$, and $\bar{z}^{3}<u_{1}^{3}$ if and only if $\alpha_{1} \alpha_{2} \alpha_{3}<1, \rho_{3}=\alpha_{1} \rho_{1}$, and $\rho_{1}$ satisfies (A.68). In this case there is a continuum of SSPEs. Any $\lambda \geq 1$ satisfying (A.66) and (A.67) induces an SSPE given by (A.57), (A.58), and (A.65). Equilibrium utilities depend on $\lambda$ and satisfy $\bar{z}^{1}=u_{2}^{1}, \bar{z}^{2}<u_{3}^{2}$, and $\bar{z}^{3}<u_{1}^{3}$.

The SSPE does not involve delay if and only if $\lambda=1$. Whenever $\rho_{1}<\frac{\alpha_{2}}{1+\alpha_{2}+\alpha_{1} \alpha_{2}}$, the lowest possible choice for $\lambda$ strictly exceeds 1 , and delay cannot be avoided.

Fully symmetric results hold for SSPEs with $\bar{p}_{m_{i}}^{i}=0, i=1,2,3, \bar{z}^{1}<u_{2}^{1}$, $\bar{z}^{2} \geq u_{3}^{2}$, and $\bar{z}^{3}<u_{1}^{3}$, and for SSPEs with $\bar{p}_{m_{i}}^{i}=0, i=1,2,3, \bar{z}^{1}<u_{2}^{1}, \bar{z}^{2}<u_{3}^{2}$, and $\bar{z}^{3} \geq u_{1}^{3}$.

\section{A.4.4 Case 4}

It holds that $(\bar{p}, \bar{a}) \in P \times A$ is an SSPE if and only if there is $\bar{\pi}$ such that

$$
\begin{aligned}
& \bar{p}=\left[\begin{array}{lll}
1 & 0 & 0 \\
0 & 1 & 0 \\
0 & 0 & 1
\end{array}\right] \\
& \bar{a}^{1}=\left[\begin{array}{l}
1 \\
1 \\
0
\end{array}\right], \bar{a}^{2}=\left[\begin{array}{l}
0 \\
1 \\
1
\end{array}\right], \text { and } \bar{a}^{3}=\left[\begin{array}{l}
1 \\
0 \\
1
\end{array}\right], \\
& \bar{\pi}_{1} u_{1}^{1}+\bar{\pi}_{2} u_{2}^{1}+\bar{\pi}_{3} u_{3}^{1}<u_{2}^{1}, \\
& \bar{\pi}_{1} u_{1}^{2}+\bar{\pi}_{2} u_{2}^{2}+\bar{\pi}_{3} u_{3}^{2}<u_{3}^{2}, \\
& \bar{\pi}_{1} u_{1}^{3}+\bar{\pi}_{2} u_{2}^{3}+\bar{\pi}_{3} u_{3}^{3}<u_{1}^{3}, \\
& \bar{\pi}_{1}+\bar{\pi}_{2}+\bar{\pi}_{3}=1, \\
& \bar{\pi}_{1}: \bar{\pi}_{2}: \bar{\pi}_{3}=\rho_{1}: \rho_{2}: \rho_{3} .
\end{aligned}
$$

The equalities in (A.74) immediately lead to the conclusion that $\bar{\pi}_{1}=\rho_{1}, \bar{\pi}_{2}=\rho_{2}$, and $\bar{\pi}_{3}=\rho_{3}$. The inequalities in (A.71)-(A.73) are equivalent to the following conditions:

$$
\begin{aligned}
& \frac{\rho_{3}}{\rho_{1}}>\alpha_{1}, \\
& \frac{\rho_{1}}{\rho_{2}}>\alpha_{2}, \\
& \frac{\rho_{2}}{\rho_{3}}>\alpha_{3} .
\end{aligned}
$$


It is immediate to verify that (A.75)-(A.77) imply that $\alpha_{1} \alpha_{2} \alpha_{3}<1$.

By definition of the case, the equilibrium utilities satisfy $\bar{z}^{1}<u_{m_{1}}^{1}, \bar{z}^{2}<u_{m_{2}}^{2}$ and $\bar{z}^{3}<u_{m_{3}}^{3}$. Since each player accepts his middle alternative for sure, the conditional equilibrium utilities satisfy $\bar{v}_{1}^{1}=u_{1}^{1}, \bar{v}_{2}^{2}=u_{2}^{2}$ and $\bar{v}_{3}^{3}=u_{3}^{3}$. Therefore, each player has an advantage to propose and, as the recognized player, each player can realize his best alternative for sure.

We summarize our findings in the following theorem.

Theorem A.4.4 There is an $\operatorname{SSPE}(\bar{p}, \bar{a}) \in P \times A$ with $\bar{p}_{m_{i}}^{i}=0, i=1,2,3, \bar{z}^{1}<$ $u_{2}^{1}, \bar{z}^{2}<u_{3}^{2}$, and $\bar{z}^{3}<u_{1}^{3}$ if and only if $\alpha_{1} \alpha_{2} \alpha_{3}<1$ and $\rho$ is such that (A.75)-(A.77) are satisfied. In this case there is a unique SSPE. It is given by (A.69) and (A.70). The equilibrium utilities satisfy $\bar{z}^{1}<u_{2}^{1}, \bar{z}^{2}<u_{3}^{2}$, and $\bar{z}^{3}<u_{1}^{3}$.

\section{Appendix B}

\section{Proof of Theorem 4.2:}

$\Rightarrow$ After observing that $\sum_{j=0}^{3} p_{j}^{h} r_{j h}^{h-1} r_{j h}^{h+1}<1$ for some player $h$, i.e. player $h$ makes with positive probability a proposal that is accepted with positive probability is equivalent to $\pi_{0}=0$, this direction follows from the derivations in Sect. 4.

$\Leftarrow$ We first argue that a solution $(p, r, v, z)$ to (4.1)-(4.7) corresponds to a strategy profile $(p, r)$ inducing utilities $(v, z)$ and satisfying the one-shot deviation property. To show that $(v, z)$ are the utilities induced by $(p, r)$ we have to show that given the strategy profile $(p, r)$ the system (4.1)-(4.2) has a unique solution. We substitute the expression for $v_{h}^{i}$ given in (4.1) in (4.2) and obtain a system of three equations and three unknowns of the form

$$
z^{i}=c^{i}+d z^{i}, \quad i=1,2,3
$$

The constant $d$ is given by

$$
d=\sum_{h=1}^{3} \rho_{h} \sum_{j=0}^{3} p_{j}^{h} r_{j h}^{h-1} r_{j h}^{h+1}<1,
$$

where the inequality follows from the fact that $\rho_{h}$ is positive for every player $h$. Since $d<1$, the uniqueness of $z$ follows immediately, leading to the uniqueness of $v$. Now it follows from (4.1)-(4.7) that $(p, r)$ has the one-shot deviation property.

We argue next that the absence of a profitable one-shot deviation implies the absence of a profitable deviation, proving that $(p, r)$ is an SSPE. The usual proofs do not apply because future payoffs are not discounted. Nevertheless, the property that for some $h$ it holds that $\sum_{j=0}^{3} p_{j}^{h} r_{j h}^{h-1} r_{j h}^{h+1}<1$ coupled with the observation that $\rho_{h}>0$ implies that every round there is a positive probability that negotiations terminate. Suppose there is some player, say $i$, who has a profitable deviation from $(p, r)$ at some decision node. The feature that every round there is a positive probability that negotiations terminate implies that player $i$ also has a profitable deviation from $(p, r)$ 
that coincides with the strategy prescribed by $\left(p^{i}, r^{i}\right)$ except for a finite number of decision nodes, exactly as in the case with discounting. Finally, the usual backwards induction argument shows that player $i$ must then also have a profitable one-shot deviation.

Proof of Theorem 4.3: We show first that each player $i$ has $z^{i}$ strictly exceeding the utility $u_{w_{i}}^{i}$ of his worst alternative. Suppose, on the contrary, that player $i$ has $z^{i}=u_{w_{i}}^{i}$. The probability is therefore 1 that the alternative $x_{w_{i}}$ is accepted at some point in time, since otherwise the utility of $i$ strictly exceeds $u_{w_{i}}^{i}$. Therefore, it follows that $z^{i-1}=u_{b_{i-1}}^{i-1}$ and $z^{i+1}=u_{m_{i+1}}^{i+1}$. Since $u_{m_{i}}^{i-1}=u_{w_{i-1}}^{i-1}<u_{b_{i-1}}^{i-1}=z^{i-1}$, (4.7) yields that player $i-1$ rejects proposal $x_{m_{i}}$ by player $i$ with probability 1 , so $r_{m_{i} i}^{i-1}=1$. Since $r_{m_{i} i}^{i-1}=1$ and $z^{i+1}=u_{m_{i+1}}^{i+1}<u_{b_{i+1}}^{i+1}=u_{m_{i}}^{i+1}$, (4.4) yields that player $i+1$ rejects proposal $x_{m_{i}}$ by player $i$ with probability 0 . Proposal $x_{m_{i}}$ by player $i$ is therefore accepted with probability 1 , so $v_{i}^{i} \geq u_{m_{i}}^{i}>u_{w_{i}}^{i}$. Since $v_{i-1}^{i} \geq u_{w_{i}}^{i}$ and $v_{i+1}^{i} \geq u_{w_{i}}^{i}$, we find that $u_{w_{i}}^{i}=z^{i}=\rho_{1} v_{1}^{i}+\rho_{2} v_{2}^{i}+\rho_{3} v_{3}^{i}>u_{w_{i}}^{i}$, a contradiction. We conclude that each player has $z^{i}$ strictly exceeding $u_{w_{i}}^{i}$, i.e.

$$
z^{i}>u_{w_{i}}^{i}, \quad i=1,2,3 .
$$

We show next that each player $i$ has $z^{i}$ strictly lower than the utility of his best alternative, $u_{b_{i}}^{i}$. If some player $i$ has $z^{i}=u_{b_{i}}^{i}$, then alternative $x_{b_{i}}$ is accepted with probability 1 , so $z^{i+1}=u_{b_{i}}^{i+1}=u_{w_{i+1}}^{i+1}$, a contradiction to (4.12). We have found that

$$
z^{i}<u_{b_{i}}^{i}, \quad i=1,2,3
$$

Next, we argue that any voter rejects his worst alternative for sure. To see this, when player $h$ proposes alternative $x_{w_{i}}, i \neq h$, then player $i$ is the last one to vote. It holds by (4.12) that $z^{i}>u_{w_{i}}^{i}$, so by (4.7), $r_{w_{i} h}^{i}=1$. We have shown that

$$
r_{w_{i} h}^{i}=1, \quad i=1,2,3, h \neq i
$$

We continue by establishing that, independent of who proposes, the recognized player can always conclude the bargaining for sure by proposing either his worst or his middle alternative. Consider a proposal $x_{m_{i}}$ by player $i$, so player $i$ proposes his middle alternative and player $i+1$, for whom this is the best alternative, votes before player $i-1$. We argue that this proposal will be accepted with probability 1 by player $i+1$, i.e. $r_{m_{i} i}^{i+1}=0$. By (4.9), since $m_{i}=w_{i-1}, r_{m_{i} i}^{i-1}=1$. Using that $m_{i}=b_{i+1}$, we know by (4.13), $u_{m_{i}}^{i+1}>z^{i+1}$. Since $r_{m_{i} i}^{i-1}=1$, we use (4.4) to conclude that $r_{m_{i} i}^{i+1}=0$. We have derived that

$$
r_{21}^{2}=r_{32}^{3}=r_{13}^{1}=0 \text {. }
$$

Consider now a proposal $x_{w_{i}}$ by player $i$ meaning player $i$ proposes his worst alternative. We argue that this proposal will be accepted with probability one, i.e. 
$r_{w_{i} i}^{i-1} r_{w_{i} i}^{i+1}=0$. Since $w_{i}=b_{i-1}$, it follows from (4.13) that $u_{w_{i}}^{i-1}>z^{i-1}$, so by (4.4), $r_{w_{i} i}^{i-1}=0$ or $r_{w_{i} i}^{i+1}=0$. We have derived that

$$
r_{31}^{2} r_{31}^{3}=r_{12}^{1} r_{12}^{3}=r_{23}^{1} r_{23}^{2}=0
$$

As a corollary, a recognized player will never propose his worst alternative, because he can do strictly better by proposing his middle alternative, i.e.

$$
p_{w_{i}}^{i}=0, \quad i=1,2,3 .
$$

We have already argued that each $z^{i}$ is a weighted average of $u_{j}^{i}, j=1,2,3$, with weights $\pi_{j}$ independent of $i$. We argue next that all these weights are positive. If only one weight would be positive, we would get a contradiction to (4.12) for some $i$. Suppose that exactly two weights are positive, without loss of generality the weights $\pi_{1}$ on outcome $x_{1}$ and $\pi_{2}$ on $x_{2}$ sum up to one and $\pi_{3}=0$, so $z^{i}=\pi_{1} u_{1}^{i}+\pi_{2} u_{2}^{i}$. From (4.4) and (4.9), the proposal $x_{1}$ by player 1 is accepted with probability 1 by player 3 . The proposal $x_{2}$ by player 1 is accepted with probability 1 according to (4.10). We can now use (4.3) to conclude that $p_{0}^{1}=p_{2}^{1}=0$, and since $p_{3}^{1}=0$ by (4.8), we know that $p_{1}^{1}=$ 1. A proposal $x_{2}$ by player 2 would be rejected with probability 1 by player 1 using (4.5) and the fact that $r_{22}^{3}=1$ by (4.9). Player 3 never proposes $x_{2}$ by (4.8). It now follows that $\pi_{1}=1$, a contradiction to (4.12). We conclude that all weights are positive,

$$
\pi_{1}, \pi_{2}, \pi_{3}>0 .
$$

Proof of Theorem 4.4: Assume that $(p, r) \in P \times R$ satisfies (4.1)-( 4.7). We show that $(p, \bar{r})$ satisfies (4.1)-(4.7), where $\bar{r}$ is as defined in Theorem 4.4. We verify first that $\bar{r}$ is proposer independent. Indeed, for $i=1,2,3$, we have the following. It holds by definition that $\bar{r}_{0 h}^{i}=1, h \neq i$. We have by definition that $\bar{r}_{b_{i} i+1}^{i}=0$ and $\bar{r}_{b_{i} i-1}^{i}=r_{b_{i} i-1}^{i}=0$, where the last equality holds by (4.10). Also it holds by definition that $\bar{r}_{m_{i} i+1}^{i}=r_{m_{i} i+1}^{i}$ and $\bar{r}_{m_{i} i-1}^{i}=r_{m_{i} i+1}^{i}$, so $\bar{r}_{m_{i} i+1}^{i}=\bar{r}_{m_{i} i-1}^{i}$. Finally, we have $r_{w_{i} h}^{i}=1, h \neq i$, by (4.9), and $\bar{r}_{w_{i} h}^{i}=r_{w_{i} h}^{i}, h \neq i$, by definition.

We show next that

$$
r_{j h}^{h-1} r_{j h}^{h+1}=\bar{r}_{j h}^{h-1} \bar{r}_{j h}^{h+1}, \quad h=1,2,3, j=0,1,2,3 .
$$

For $j=0$, this follows immediately from the definition of $R$. Three possible cases remain: (i) $j=b_{h}$, (ii) $j=m_{h}$, and (iii) $j=w_{h}$.

Case (i), $j=b_{h}$. Since $b_{h}=m_{h-1}=w_{h+1}$, we have

$$
\bar{r}_{b_{h} h}^{h-1} \bar{r}_{b_{h} h}^{h+1}=\bar{r}_{m_{h-1} h}^{h-1} \bar{r}_{w_{h+1} h}^{h+1}=r_{m_{h-1} h}^{h-1} r_{w_{h+1} h}^{h+1}=r_{b_{h} h}^{h-1} r_{b_{h} h}^{h+1}
$$

where the second equality follows by definition of $\bar{r}$. 
Case (ii), $j=m_{h}$. Since $m_{h}=w_{h-1}=b_{h+1}$, we obtain

$$
\bar{r}_{m_{h} h}^{h-1} \bar{r}_{m_{h} h}^{h+1}=\bar{r}_{w_{h-1} h}^{h-1} \bar{r}_{b_{h+1} h}^{h+1}=r_{w_{h-1} h}^{h-1} r_{b_{h+1} h}^{h+1}=r_{m_{h} h}^{h-1} r_{m_{h} h}^{h+1},
$$

where the second equality follows by definition of $\bar{r}$.

Case (iii), $j=w_{h}$. By (4.11), it holds that $r_{w_{h} h}^{h-1} r_{w_{h} h}^{h+1}=0$. Since $w_{h}=b_{h-1}$, we have that $\bar{r}_{w_{h} h}^{h-1}=\bar{r}_{b_{h-1} h}^{h-1}=0$, where the last equality follows by definition of $\bar{r}$. It follows that $\bar{r}_{w_{h} h}^{h-1} \bar{r}_{w_{h} h}^{h+1}=0$.

Using (B.1) we have that $(p, \bar{r}, v, z)$ satisfies (4.1), (4.2), and (4.3). We verify next that $(p, \bar{r}, v, z)$ satisfies (4.4)-(4.7). Consider some $\bar{r}_{j h}^{i}$. If $j=b_{i}$, then $\bar{r}_{j h}^{i}=0$ and $i=f_{j h}$, so (4.4), (4.6), and (4.7) hold trivially. Implication (4.5) holds as well, since $z^{i}<u_{b_{i}}^{i}$ by (4.13). If $j=w_{i}$, then $\bar{r}_{j h}^{i}=r_{j h}^{i}=1$ by (4.9), so (4.5) and (4.7) hold trivially. Since by (4.12) $z^{i}>u_{w_{i}}^{i}$, we find that (4.4) and (4.6) also hold. If $j=m_{i}$ and $h=i+1$, then $i=f_{j h}$, so (4.6) and (4.7) hold trivially. We have that $\bar{r}_{j h}^{i}=r_{j h}^{i}$ and $\bar{r}_{j h}^{i^{\prime}}=\bar{r}_{w_{i-1} i+1}^{i-1}=r_{w_{i-1} i+1}^{i-1}=r_{j h}^{i^{\prime}}$, so (4.4) and (4.5) hold. Finally, we consider the case where $j=m_{i}$ and $h=i-1$, so $i=s_{j h}$ and (4.4) and (4.5) hold trivially. Assume $\bar{r}_{j h}^{i}>0$. Since by definition $\bar{r}_{j h}^{i}=\bar{r}_{m_{i} i-1}^{i}=r_{m_{i} i+1}^{i}$, we have $r_{m_{i} i+1}^{i}>0$, so by (4.4) $z^{i} \geq u_{m_{i}}^{i}$ or $r_{m_{i} i+1}^{i-1}=0$. Since $m_{i}=w_{i-1}$, (4.9) implies $r_{m_{i} i+1}^{i-1}=1$, so $z^{i} \geq u_{m_{i}}^{i}$. It follows that (4.6) holds. Assume $\bar{r}_{j h}^{i}<1$. Since by definition $\bar{r}_{j h}^{i}=\bar{r}_{m_{i} i-1}^{i}=r_{m_{i} i+1}^{i}$, we have $r_{m_{i} i+1}^{i}<1$, so by (4.5) $z^{i} \leq u_{m_{i}}^{i}$ or $r_{m_{i} i+1}^{i-1}=0$. Since $m_{i}=w_{i-1}$, (4.9) implies $r_{m_{i} i+1}^{i-1}=1$, so $z^{i} \leq u_{m_{i}}^{i}$. It follows that (4.7) holds.

\section{Proof of Theorem 4.5:}

$(\Rightarrow)$ This direction follows immediately from the results derived in Sect. 4.

$(\Leftarrow)$ This direction follows from Theorem 4.2 by defining, for $h=1,2,3, i=1,2,3$, and $j=0,1,2,3$,

$$
\begin{aligned}
\bar{v}_{h}^{i} & =\bar{p}_{m_{h}}^{h} u_{m_{h}}^{i}+\bar{p}_{b_{h}}^{h} \bar{a}_{b_{h}}^{h-1} u_{b_{h}}^{i}+\bar{p}_{b_{h}}^{h}\left(1-\bar{a}_{b_{h}}^{h-1}\right) \bar{z}^{i}, \\
\bar{r}_{j h}^{i} & =1-\bar{a}_{j}^{i},
\end{aligned}
$$

and verifying that a solution $(\bar{p}, \bar{a}, \bar{\pi}, \bar{z})$ to (4.15)-(4.22) inducing expected utilities $\bar{v}$ and rejection probabilities $\bar{r}$ leads to a solution $(\bar{p}, \bar{r}, \bar{v}, \bar{z})$ to (4.1)-(4.7) with $\sum_{j=0}^{3} \bar{p}_{j}^{h} \bar{r}_{j h}^{h-1} \bar{r}_{j h}^{h+1}<1$.

Proof of Theorem 6.1: Necessity follows by Table 3. We now turn to sufficiency of the condition. By Theorem A. 1 an SSPE exists if $\alpha_{1} \alpha_{2} \alpha_{3}=1$. It remains to be shown that an SSPE exists if $\alpha_{1} \alpha_{2} \alpha_{3}<1$.

By Theorem A.4.4 an SSPE exists if $\rho_{1} / \rho_{2}>\alpha_{2}, \rho_{2} / \rho_{3}>\alpha_{3}$, and $\rho_{3} / \rho_{1}>\alpha_{1}$. Consider now the cases where the conditions of Theorem A.4.4 are not satisfied. We claim that then

$$
\left(\frac{\rho_{3}}{\rho_{1}} \geq \alpha_{1} \text { and } \frac{\rho_{1}}{\rho_{2}} \leq \alpha_{2}\right) \text { or }\left(\frac{\rho_{1}}{\rho_{2}} \geq \alpha_{2} \text { and } \frac{\rho_{2}}{\rho_{3}} \leq \alpha_{3}\right) \text { or }\left(\frac{\rho_{2}}{\rho_{3}} \geq \alpha_{3} \text { and } \frac{\rho_{3}}{\rho_{1}} \leq \alpha_{1}\right) \text {. }
$$


Indeed, assume, without loss of generality, $\rho_{1} / \rho_{2} \leq \alpha_{2}$. Either it holds that $\rho_{3} / \rho_{1} \geq \alpha_{1}$ or $\rho_{3} / \rho_{1}<\alpha_{1}$. In the former case the first formula in (B.2) is true, in the latter case it should hold that $\rho_{2} / \rho_{3} \geq \alpha_{3}$, since otherwise

$$
1=\frac{\rho_{1}}{\rho_{2}} \frac{\rho_{2}}{\rho_{3}} \frac{\rho_{3}}{\rho_{1}}<\alpha_{2} \alpha_{3} \alpha_{1}<1
$$

and the third formula in (B.2) is true.

We show next that an SSPE exists whenever $\rho_{3} / \rho_{1} \geq \alpha_{1}$ and $\rho_{1} / \rho_{2} \leq \alpha_{2}$. The other two cases in (B.2) follow by symmetry. If $\rho_{3} / \rho_{1}=\alpha_{1}$ and $\rho_{1} / \rho_{2} \leq \alpha_{2}$, then line 1 in Table 3 corresponding to Theorem A.4.3 implies the existence of an SSPE since $\rho_{1} / \rho_{2} \leq \alpha_{2}$ implies $\rho_{1}<1 /\left(1+\alpha_{1}+\alpha_{1} \alpha_{3}\right)$. Suppose, by contradiction, that $\rho_{1} \geq 1 /\left(1+\alpha_{1}+\alpha_{1} \alpha_{3}\right)$. Then

$$
\begin{aligned}
1 & =\rho_{1}+\rho_{2}+\rho_{3} \geq \frac{1}{1+\alpha_{1}+\alpha_{1} \alpha_{3}}+\frac{1}{\alpha_{2}+\alpha_{1} \alpha_{2}+\alpha_{1} \alpha_{2} \alpha_{3}}+\frac{\alpha_{1}}{1+\alpha_{1}+\alpha_{1} \alpha_{3}} \\
& =\frac{1+\alpha_{2}+\alpha_{1} \alpha_{2}}{\alpha_{2}+\alpha_{1} \alpha_{2}+\alpha_{1} \alpha_{2} \alpha_{3}}>1,
\end{aligned}
$$

a contradiction. If $\rho_{1} / \rho_{2}=\alpha_{2}$ and $\rho_{3} / \rho_{1}>\alpha_{1}$, then line 2 in Table 3 corresponding to Theorem A.4.3 implies the existence of an SSPE since $\rho_{3} / \rho_{1} \geq \alpha_{1}$ implies $\rho_{2}<$ $1 /\left(1+\alpha_{2}+\alpha_{1} \alpha_{2}\right)$. Suppose, by contradiction, that $\rho_{2} \geq 1 /\left(1+\alpha_{2}+\alpha_{1} \alpha_{2}\right)$. Then

$$
1=\rho_{1}+\rho_{2}+\rho_{3}>\frac{\alpha_{2}}{1+\alpha_{2}+\alpha_{1} \alpha_{2}}+\frac{1}{1+\alpha_{2}+\alpha_{1} \alpha_{2}}+\frac{\alpha_{1} \alpha_{2}}{1+\alpha_{2}+\alpha_{1} \alpha_{2}}=1
$$

a contradiction.

It remains to be shown that an SSPE exists if $\rho_{3} / \rho_{1}>\alpha_{1}$ and $\rho_{1} / \rho_{2}<\alpha_{2}$. By line 1 in Table 3 corresponding to Theorem A.4.2, an SSPE exists if $\rho_{3} / \rho_{1} \geq \alpha_{1}$, $\rho_{1} / \rho_{2} \leq \alpha_{2}$, and $\rho_{1} / \rho_{3} \geq \beta_{1}$, and by line 1 in Table 3 corresponding to Theorem A.3.1 an SSPE exists if $\rho_{1} / \rho_{3}<\beta_{1}$ and $\rho_{2} \geq \alpha_{3} \beta_{3}$.

It remains to be shown that an SSPE exists if $\rho_{3} / \rho_{1}>\alpha_{1}, \rho_{1} / \rho_{2}<\alpha_{2}, \rho_{1} / \rho_{3}<\beta_{1}$, and $\rho_{2}<\alpha_{3} \beta_{3}$. This follows from line 1 in Table 3 corresponding to Theorem A.3.2.

Proof of Theorem 6.2: Existence follows from Theorem 6.1. Leave out the games satisfying the conditions of Theorems A.1, A.4.1, and A.4.3. This corresponds to a set of games whose closure has Lebesgue measure zero. Comparing the conditions in any two distinct rows (that do not correspond to Theorem A.3.1) of Table 3 leads to the conclusion that the corresponding two sets of parameters have an empty intersection. This conclusion follows directly in most cases. In some cases one has to make use of the property that $\alpha_{i} \beta_{i}<1$, which implies that we cannot have simultaneously $\rho_{j} / \rho_{k}<\alpha_{i}$ and $\rho_{k} / \rho_{j}<\beta_{i}$. Finally, each of the Theorems A.3.1, A.3.2, A.4.2, and A.4.4 identify a unique SSPE. 
Proof of Theorem 7.1: First we consider the case where $\alpha_{1} \alpha_{2} \alpha_{3}=1$. From Table 4 and Theorem A.1 it follows that we can choose

$$
\rho_{1}=\frac{1}{1+\alpha_{2}+\alpha_{1} \alpha_{2}}, \quad \rho_{2}=\frac{\alpha_{1} \alpha_{2}}{1+\alpha_{2}+\alpha_{1} \alpha_{2}}, \quad \text { and } \quad \rho_{3}=\frac{\alpha_{2}}{1+\alpha_{2}+\alpha_{1} \alpha_{2}},
$$

which leads to a delay probability of $1-1=0$.

Next we consider the case where $\alpha_{1} \alpha_{2} \alpha_{3}<1$. We show that $\rho$ can be chosen such that the conditions of Theorem A.4.4 as listed in Table 3 are satisfied, which demonstrates the absence of delay. We define $\gamma=1 / \sqrt[3]{\alpha_{1} \alpha_{2} \alpha_{3}}>1$ and

$$
\begin{aligned}
& \rho_{1}=\frac{\gamma^{2} \alpha_{2} \alpha_{3}}{1+\gamma \alpha_{3}+\gamma^{2} \alpha_{2} \alpha_{3}}, \quad \rho_{2}=\frac{\gamma \alpha_{3}}{1+\gamma \alpha_{3}+\gamma^{2} \alpha_{2} \alpha_{3}}, \quad \text { and } \\
& \rho_{3}=\frac{1}{1+\gamma \alpha_{3}+\gamma^{2} \alpha_{2} \alpha_{3}} .
\end{aligned}
$$

It therefore holds that

$$
\frac{\rho_{1}}{\rho_{2}}=\gamma \alpha_{2}>\alpha_{2}, \quad \frac{\rho_{2}}{\rho_{3}}=\gamma \alpha_{3}>\alpha_{3}, \quad \text { and } \quad \frac{\rho_{3}}{\rho_{1}}=\frac{1}{\gamma^{2} \alpha_{2} \alpha_{3}}=\gamma \alpha_{1}>\alpha_{1} .
$$

Proof of Theorem 7.2: Without loss of generality, we may assume that for all $n \in$ $\mathbb{N},\left(u, \rho^{n}\right)$ satisfies the conditions of exactly one of the theorems, i.e. one of the 15 subcases displayed in Table 4.

According to Table 4, the lower bound on the delay probability following from an SSPE of Theorem A.1 is given by

$$
1-\left(1+\alpha_{2}+\alpha_{1} \alpha_{2}\right) \min \left\{\rho_{1}^{n}, \frac{\rho_{2}^{n}}{\alpha_{1} \alpha_{2}}, \frac{\rho_{3}^{n}}{\alpha_{2}}\right\} \text {. }
$$

Clearly, this lower bound converges to 1 when $n \rightarrow \infty$.

According to Table 3, the first line of conditions in Theorem A.3.1 states that $\rho_{2}^{n} \geq \alpha_{3} \beta_{3}$, so $\lim _{n \rightarrow \infty} \rho_{2}^{n}=1$. Then Table 4, first line corresponding to A.3.1, yields that $\lim _{n \rightarrow \infty} \delta_{n}=1$. The other cases corresponding to Theorem A.3.1 follow by symmetry.

According to Table 3, the first line of conditions in Theorem A.3.2 states that $\rho_{2}^{n}<$ $\alpha_{3} \beta_{3}$. Since $\alpha_{3} \beta_{3}<1$, it is impossible that $\lim _{n \rightarrow \infty} \rho_{2}^{n}=1$, so $\lim _{n \rightarrow \infty} \rho_{2}^{n}=0$. Then Table 4, first line corresponding to A.3.2, yields that $\lim _{n \rightarrow \infty} \delta_{n}=1$. The other cases corresponding to Theorem A.3.2 follow by symmetry.

It is evident from Table 4 that the delay probability following from Theorem A.4.1 goes to 1 when $n$ goes to infinity.

According to Table 3, the first line of conditions in Theorem A.4.2 states that $\rho_{1}^{n} \leq \alpha_{2} \rho_{2}^{n}$, so $\lim _{n \rightarrow \infty} \rho_{1}^{n}=0$. Then Table 4, first line corresponding to A.4.2, yields that $\lim _{n \rightarrow \infty} \delta_{n}=1$. The other cases corresponding to Theorem A.4.2 follow by symmetry. 
According to Table 3, the first line of conditions in Theorem A.4.3 states that $\rho_{1}^{n}<1 /\left(1+\alpha_{1}+\alpha_{1} \alpha_{3}\right)$, so $\lim _{n \rightarrow \infty} \rho_{1}^{n}=0$. Then Table 4 , first line corresponding to A.4.3, yields that the lower bound on the delay probability following from an SSPE converges to 1 . The other cases corresponding to Theorem A.4.3 follow by symmetry.

Table 3 demonstrates that $\left(u, \rho^{n}\right)_{n \in \mathbb{N}}$ cannot satisfy the conditions of Theorem A.4.4. Suppose without loss of generality that $\lim _{n \rightarrow \infty} \rho_{1}^{n}=0$. Since $\rho_{2}^{n}<$ $\rho_{1}^{n} / \alpha_{2}$, we have $\lim _{n \rightarrow \infty} \rho_{2}^{n}=0$. Since $\rho_{3}^{n}<\rho_{2}^{n} / \alpha_{3}$, we have $\lim _{n \rightarrow \infty} \rho_{3}^{n}=0$. It follows that $\rho$ converges to the zero vector, a contradiction.

Proof of Theorem 7.3: It is easily verified that, with the exception of Theorems A.1 and A.4.3, there is always an alternative that, in an SSPE, is never rejected when being proposed. The Conditions in Theorems A.1 and A.4.3 are only satisfied for sets of games having a closure with Lebesgue measure zero.

\section{References}

Balinski M, Laraki R (2010) Majority judgment-measuring, ranking, and electing. MIT Press, Cambridge Banks J, Duggan J (2000) A bargaining model of collective choice. Am Polit Sci Rev 94:73-88

Baron DP, Ferejohn JA (1989) Bargaining in legislatures. Am Polit Sci Rev 83:1181-1206

Bernheim BD, Rangel A, Rayo L (2006) The power of the last word in legislative policy making. Econometrica 74:1161-1190

Bhaskar V, Mailath GJ, Morris S (2013) A foundation for Markov equilibria in sequential games with finite social memory. Rev Econ Stud 80:925-948

Binmore K (1987) Perfect equilibria in bargaining models. In: Binmore K, Dasgupta P (eds) The economics of bargaining. Basil Blackwell, Oxford, pp 77-105

Bloch F (1996) Sequential formation of coalitions in games with externalities and fixed payoff division. Games Econ Behav 14:90-123

Bloch F, Diamantoudi E (2011) Noncooperative formation of coalitions in hedonic games. Int J Game Theory 40:263-280

Chatterjee K, Dutta B, Ray D, Sengupta K (1993) A noncooperative theory of coalitional bargaining. Rev Econ Stud 60:463-477

Chwe MS-Y (1994) Farsighted coalitional stability. J Econ Theory 63:299-325

Cox GW (1984) Non-collegial simple games and the nowhere denseness of the set of preference profiles having a core. Soc Choice Welf 1:159-164

Eraslan H (2002) Uniqueness of stationary equilibrium payoffs in the Baron-Ferejohn model. J Econ Theory 103:11-30

Eraslan H, McLennan A (2013) Uniqueness of stationary equilibrium payoffs in coalitional bargaining. J Econ Theory 148:2195-2222

Fink AM (1964) Equilibrium in a stochastic $n$-person game. J Sci Hiroshima Univ Ser A-I 28:89-93

Flesch J, Kuipers J, Schoenmakers G, Vrieze K (2010) Subgame perfection in positive recursive games with perfect information. Math Oper Res 35:193-207

Haller H, Lagunoff R (2000) Genericity and Markovian behavior in stochastic games. Econometrica 68:1231-1248

Harsanyi JC (1977) Rational behavior and bargaining equilibrium in games and social situations. Cambridge University Press, Cambridge

Herings PJJ, Houba H (2015) Costless delay in negotiations, Tinbergen Institute Discussion Paper 2015010/II, Amsterdam

Herings PJJ, Peeters RJAP (2004) Stationary equilibria in stochastic games: structure, selection, and computation. J Econ Theory 118:32-60

Le Breton M (1987) On the core of voting games. Soc Choice Welf 4:295-305

Maskin ES, Tirole J (2001) Markov perfect equilibrium, I. Observable actions. J Econ Theory 100:191-219

McKelvey RD (1976) Intransitivities in multidimensional voting models and some implications for agenda control. J Econ Theory 12:472-482 
McKelvey RD (1979) General conditions for global intransitivities in formal voting models. Econometrica 47:1085-1112

Plott CR (1967) A notion of equilibrium and its possibility under majority rule. Am Econ Rev 57:787-806

Roessler C, Shelegia S, Strulovici B (2013) The Roman metro problem: dynamic voting and the limited power of commitment. Working Paper, pp 1-30

Rubinstein A (1979) A note about the "nowhere denseness" of societies having an equilibrium under majority rule. Econometrica 47:511-514

Rubinstein A (1982) Perfect equilibrium in a bargaining model. Econometrica 50:97-109

Selten R (1981) A noncooperative model of characteristic function bargaining. In: Böhm V, Nachtkamp $\mathrm{HH}$ (eds) Essays in game theory and mathematical economics in honor of Oskar Morgenstern. Bibliografisches Institut, Mannheim, pp 131-151

Schofield N (1983) Generic instability of majority rule. Rev Econ Stud 50:695-705

Sobel MJ (1971) Noncooperative stochastic games. Ann Math Stud 42:1930-1935

Solan E (1999) Three-player absorbing games. Math Oper Res 24:669-698

Takahashi M (1964) Equilibrium points of stochastic, non-cooperative $n$-person games. J Sci Hiroshima Univ Ser A-I 28:95-99

Thuijsman F, Raghavan TES (1997) Perfect information stochastic games and related classes. Int J Game Theory 26:403-408

Zeuthen F (1930) Problems of monopoly and economic welfare. Routledge, London 